\title{
Quantum interference and the spin orbit interaction in mesoscopic normal-superconducting junctions
}

\author{
Keith Slevin and Jean-Louis Pichard \\ Service de Physique de l'État Condensé, CEA-Saclay, 91191 Gif-sur-Yvette, \\ France \\ Pier A. Mello \\ Instituto de Fisica, UNAM, Apartado Postal 20-364, 01000 Mexico D.F.
}

Short title: The SO interaction in mesoscopic NS junctions.

\begin{abstract}
We calculate the quantum correction to the classical conductance of a disordered mesoscopic normal-superconducting (NS) junction in which the electron spatial and spin degrees of freedom are coupled by an appreciable spin orbit interaction. We use random matrix theory to describe the scattering in the normal part of the junction and consider both quasi-ballistic and diffusive junctions. The dependence of the junction conductance on the Schottky barrier transparency at the NS interface is also considered. We find that the quantum correction is sensitive to the breaking of spin rotation symmetry even when the junction is in a magnetic field and time reversal symmetry is broken. We demonstrate that this sensitivity is due to quantum interference between scattering processes which involve electrons and holes traversing closed loops in the same direction. We explain why such processes are sensitive to the spin orbit interaction but not to a magnetic field. Finally we consider the effect of the spin orbit interaction on the phenomenon of "reflectionless tunnelling."
\end{abstract}

74.80.Fp,74.50+r,72.10.Bg

\section{INTRODUCTION}

In mesoscopic samples of disordered normal metals at low temperatures it is possible to observe a quantum correction to the classical Boltzmann conductance [1]. Here mesoscopic means that within the sample, the interaction of a single electron with other degrees of freedom, such as other electrons, phonons, magnetic impurities etc., can be neglected. Such a situation can be realised in semiconductor and metal nanostructures cooled to milli-Kelvin temperatures. The origin of the quantum correction is quantum interference between time reversed scattering paths. (By "time reversed path" we mean that the electron follows the same trajectory but in the opposite sense.) The correction is sensitive to the breaking of spin rotation symmetry and is suppressed by the breaking of time reversal symmetry [2]. The spin orbit interaction has the important property of breaking spin rotation symmetry while preserving time reversal symmetry.

As an example, the two probe conductance $G=\left(e^{2} / h\right) g$ of a quasi-one dimensional mesoscopic wire can be expressed in the form $g=g^{c l}+\delta g$ [3]. The classical conductance of the wire is $O(N): g^{c l}=N /(1+s)$ where $N$ is the number of scattering channels and $s=L / l$ is the ratio of the length $L$ of the wire to the elastic mean free path $l$. In the absence of an applied magnetic field the quantum correction $\delta g$, also called the "weak localisation correction", is $O\left(N^{0}\right)$. More exactly, in the diffusive regime $1 \ll s \ll N$ we have $\delta g=-2 / 3$ if the spin orbit interaction is negligible and $\delta g=+1 / 3$ if it is not. In a magnetic field sufficient to break time reversal symmetry the quantum correction is suppressed and becomes $O(1 / N)$.

More recently, a quantum correction to the classical conductance of a disordered mesoscopic normal superconducting (NS) junction was observed [4]. The correction is most pronounced in junctions where the transparency $\Gamma$ of the Schottky barrier at the NS interface is low $\Gamma \ll 1$ and the length of the normal part is such that $\Gamma s \simeq 1$. Under these conditions a phenomenon known as "reflectionless tunnelling" occurs. In [5] it is suggested that quantum interference is responsible for this effect. The type of scattering paths involved in this quantum interference contain a segment where the path of an electron (hole) incident on the NS boundary is subsequently retraced by an Andreev reflected hole (electron.) The enhancement of the junction conductance can be as much as several times greater than the classical conductance. 
In this paper we investigate the effect of the coupling of the spin and spatial degrees of freedom of the electrons by an appreciable spin orbit interaction has on this quantum correction. As previously mentioned, this has an important effect on the quantum correction in a normal metal. As the electron diffuses through the metal, the spin orbit coupling causes a simultaneous diffusion of the direction of its spin. Interference between different spin states is now possible and this modifies the interference responsible for the weak localisation correction. What role then, if any, does this spin diffusion play in the NS junction?

At first sight the answer to this question would be appear to be none at all. The argument is as follows: the spin rotations of an electron and an Andreev reflected hole, which traverse time reversed paths, are exactly opposite and cancel each other. Since interference between scattering processes involving such paths is believed to be responsible for the quantum correction, it should be insensitive to the spin orbit interaction. As we shall demonstrate below this is too simple and in fact the spin orbit interaction does affect the quantum correction to the classical conductance of an NS junction. We have found the flaw in the argument is that it is not only processes involving electrons and holes traversing time reversed paths which are responsible for the quantum correction. Processes in which electrons and holes traverse loops in the same sense, what we shall call here identical paths, also contribute and in this case the spin rotations do not cancel each other. The most striking consequence of the existence of interference involving such paths is that the quantum correction is sensitive to the spin orbit interaction even in a magnetic field.

In the normal metal we have seen that the quantum correction to the classical conductance is suppressed when time reversal symmetry is broken in an applied magnetic field. This is easily understood since the symmetry between time reversed paths is broken when the time reversal symmetry is broken. In the NS junction however, the contribution of identical paths is still present even in a magnetic field; the electron and hole carry opposite charges, and so the Aharonov-Bohm phases that they accumulate as they follow such trajectories cancel. Moreover, since the electron and hole undergo identical spin rotations, as they follow identical paths, this contribution is sensitive to the spin diffusion induced by the spin orbit interaction.

The paper is organised as follows. In Sections II -IV we deal with some necessary preliminaries: the Bogolubov-de Gennes equations, scattering theory and conductance formulae for the NS junction, generalising the standard theory to take into account the spin orbit interaction.

In Section V we investigate a quasi-ballistic NS junction, that is to say a junction whose length is shorter than the mean free path but long enough so that its scattering matrix is well described by a certain random phase approximation [6]. We express the junction conductance $G_{N S}$ as a sum of two terms

$$
G_{N S}=\frac{2 e^{2}}{h} g_{N S}=\frac{2 e^{2}}{h}\left(g_{N S}^{c l}+\delta g_{N S}\right)
$$

a classical conductance $g_{N S}^{c l}$ and a quantum correction $\delta g_{N S}$ due to quantum interference. We determine the quantum correction for the four ensembles listed in Table I and the results are presented in Table II. In Section VI we present a semiclassical interpretation of these results which permits us to identify the type of scattering paths which interfere to produce the quantum correction. In agreement with Ref [5], we find that there is an important contribution due to interference between processes involving electrons and holes traversing time reversed paths, but that there is also a second contribution from processes involving electrons and holes traversing identical paths. We explain why this contribution is sensitive to the breaking of spin rotation symmetry but not to the breaking of time reversal symmetry.

After reaching a firm understanding of the quasi-ballistic junction we consider the diffusive regime, looking first at junctions without a Schottky barrier at the NS interface in Section VII. In zero magnetic field the quantum correction is known to be of $O\left(N^{0}\right)$ [7-9]. In a magnetic field the authors of Ref [10] find that the correction though smaller is still $O\left(N^{0}\right)$. The effect of the spin orbit interaction was not considered in Ref [10] and so we repeat their calculation taking it into account. We find that breaking spin rotation symmetry multiplies the correction by a factor of minus one half, regardless of whether time reversal symmetry is broken or not.

In Section VIII we discuss the dramatic enhancement of the junction conductance, known as "reflectionless tunnelling," which is observable when $\Gamma \ll 1$ and $\Gamma s \simeq 1$. Under these conditions the contribution of time reversed paths is $O(N)$ and dominates that of identical paths which is $O\left(N^{0}\right)$ so that the reflectionless tunnelling effect is, in a first approximation, insensitive to the spin orbit interaction. This conclusion has been confirmed by carrying out a numerical simulation of a junction under the relevant conditions.

\section{BOGOLUBOV-DE GENNES EQUATIONS AND THE SPIN ORBIT INTERACTION}

The Bogolubov-de Gennes (BdG) equations [11] appropriate for a metal where the electrons' spatial and spin degrees of freedom are coupled by a significant spin-orbit interaction, are 


$$
\left[\begin{array}{cc}
H_{e}, & \Delta \\
\Delta^{*}, & \mathcal{T} H_{e} \mathcal{T}
\end{array}\right]\left[\begin{array}{l}
\psi^{e} \\
\psi^{h}
\end{array}\right]=\epsilon\left[\begin{array}{l}
\psi^{e} \\
\psi^{h}
\end{array}\right]
$$

where

$$
H_{e}=H_{0}-E_{F}+\Omega
$$

Here $H_{0} \equiv H_{0}(\vec{r}, \sigma, \mu)$ is the single electron Hamiltonian of the metal incorporating the spin-orbit interaction and $E_{F}$ is the Fermi energy. In deriving this equation, it is assumed that an attractive point like interaction exists between the electrons:

$$
V\left(\vec{r}_{1}, \vec{r}_{2}\right)=-\frac{1}{2} V_{0} \delta\left(\vec{r}_{1}-\vec{r}_{2}\right)
$$

The full interacting Hamiltonian is reduced to an effective non-interacting Hamiltonian by introducing effective potentials

$$
\begin{gathered}
\Delta(\vec{r})=V_{0} \overline{\Psi(\vec{r}, \uparrow) \Psi(\vec{r}, \downarrow)} \\
\Omega(\vec{r}, \sigma, \mu)=V_{0}\left(1-2 \delta_{\sigma, \mu}\right) \overline{\Psi(\vec{r}, \sigma) \Psi^{\dagger}(\vec{r}, \mu)}
\end{gathered}
$$

where the overline indicates a thermal average with respect to the Fermi distribution function and $\Psi$ is the usual field operator appearing in the second quantised formulation of the interacting electron problem. The time reversal operator $\mathcal{T}$, which appears in the BdG equations has the form

$$
\begin{gathered}
\mathcal{T}=\rho C \\
\rho=i \sigma_{y}=\left(\begin{array}{cc}
0 & +1 \\
-1 & 0
\end{array}\right)
\end{gathered}
$$

with $C$ the operation of complex conjugation. The eigenstates of the BdG equation describe the excitations of the interacting electron system. The meaning of the electron $\psi^{e}$ and $\psi^{h}$ wavefunctions can be seen by writing the field operator as

$$
\Psi(\vec{r}, \sigma)=\sum_{n}\left[\psi_{n}^{e}(\vec{r}, \sigma) \gamma_{n}-\mathcal{T}\left[\psi_{n}^{h}(\vec{r}, \sigma)\right] \gamma_{n}^{\dagger}\right]
$$

where $\gamma_{n}$ is the annihilation operator of the excitation labelled $n$. The corresponding eigenvalue $\epsilon_{n}$ of the BdG equations corresponds to the energy of the excitation. With the aid of (2.7) the occupation of the eigenstates of $H_{e}$ for a given excitation can be determined.

\section{SCATTERING THEORY FOR THE NS JUNCTION}

In this section we develop the scattering theory appropriate to the normal superconducting junction shown schematically in Figure 1. We treat the scattering at the NS interface within Andreev's approximation. We suppose that any impurities in the system are in the region indicated by shading in Figure 1, and we make the approximation that the magnetic field is zero everywhere except in this disordered region. This is reasonable for the low fields of interest which affect the interference between electrons and holes in the normal part of the junction.

\section{A. Scattering matrices for electrons and holes}

To facilitate the explanation of the formalism, it is helpful to develop the scattering theory for a definite model. We have chosen a lattice tight binding model, the same model which we will use later in numerical simulations. An exact analogous explanation is also possible for a continuum model.

We consider a cubic lattice and take into account nearest neighbour interactions only. We denote by $\psi^{e}(x, y, z, \sigma)$ the amplitude that the electron is in an s- orbital at $(x, y, z)$ with spin $\sigma$ and similarly $\psi^{h}(x, y, z, \sigma)$ for a hole. We include in the Hamiltonian a spin orbit term which arises from the Zeeman coupling of the electron spin with the 
effective magnetic field felt by the electron as it moves in the spatially varying potential of the lattice. We ignore the direct Zeeman coupling of the spin to any external magnetic field. A simple calculation shows that the Hamiltonian has the form:

$$
\begin{array}{ll}
<x, y, z, \sigma\left|H_{e}\right| x, y, z, \mu> & E_{0} \delta_{\sigma, \mu} \\
<x, y, z, \sigma\left|H_{e}\right| x-1, y, z, \mu>= & v_{\sigma, \mu}^{x} \\
<x, y, z, \sigma\left|H_{e}\right| x, y-1, z, \mu>= & v_{\sigma, \mu}^{y} \\
<x, y, z, \sigma\left|H_{e}\right| x, y, z-1, \mu>=\exp (-i \alpha x) & v_{\sigma, \mu}^{z}
\end{array}
$$

where

$$
\begin{aligned}
v_{\sigma, \mu}^{x} & =V_{0} \delta_{\sigma, \mu}-V_{1} i\left[\sigma_{x}\right]_{\sigma, \mu} \\
v_{\sigma, \mu}^{y} & =V_{0} \delta_{\sigma, \mu}-V_{1} i\left[\sigma_{y}\right]_{\sigma, \mu} \\
v_{\sigma, \mu}^{z} & =V_{0} \delta_{\sigma, \mu}-V_{1} i\left[\sigma_{z}\right]_{\sigma, \mu}
\end{aligned}
$$

An external magnetic field $B$, applied in the $+y$ direction is modelled by Peierl's factors in the matrix elements between nearest neighbours in the $z$ direction. If $a$ is lattice constant $\alpha=2 \pi B a^{2} / \phi_{0}$ where $\phi_{0}=h / e$ is the flux quantum. The transverse dimensions are $1 \leq x \leq L_{x}$ and $1 \leq y \leq L_{y}$. The Hamiltonian (3.1) can be regarded as a three dimensional generalisation of that proposed in Ref. [12] as a model for a two dimensional electron gas formed at the surface of a III-V semiconductor.

The relevant energy scale of the model is determined by $V_{0}^{2}+V_{1}^{2}$. For convenience we shall set this to unity with the choice

$$
V_{0}=\cos \theta \quad V_{1}=\sin \theta
$$

Varying the angle $\theta$, we may set an arbitrary ratio of normal potential coupling $V_{0}$ to spin orbit coupling $V_{1}$, while keeping the extent in energy of the density of states roughly constant. With this choice, $v^{x}, v^{y}$ and $v^{z}$ are all elements of $S U(2)$. Using the homomorphism of $S U(2)$ with the three dimensional rotation group $S O(3)$, we can interpret the $v$ 's as rotations of the spin of the electron as it moves between nearest neighbours [13]. A product of nearest neighbour matrix elements along a path will have the form $\exp (i \Phi) v$ where $\Phi$ is the Aharonov Bohm phase picked up by the electron, and $v \in S U(2)$ is the rotation of the electron's spin as it traverses the path.

The Hamiltonian $H_{e}$ has the form given in (3.1) everywhere except in the disordered region located in $0<z<L$. There, some or all of the Hamiltonian matrix elements are supposed random. In Ref. [12] the diagonal elements were assumed to be independently and identically distributed, while the parameter $\theta$ controlling the spin orbit interaction was held fixed. In Refs. $[14,15]$ a random spin orbit interaction was also considered. For the present purpose we do not need to specify the precise distribution.

First, we consider the scattering of electrons incident at an energy $E=E_{F}+\epsilon$. To the left of the disordered section we expand the electron wavefunction $\psi^{e}$ in terms of the Bloch states of (3.1) with energy $E$.

$$
\begin{gathered}
\psi^{e}(x, y, z, \sigma)=\sum_{n ; \operatorname{Im} k_{n} \leq 0} a_{+n} \psi_{+n}(x, y, \sigma) \exp \left(+i k_{n} z\right)+\sum_{n ; \operatorname{Im} k_{n}=0} a_{-n} \psi_{-n}(x, y, \sigma) \exp \left(-i k_{n} z\right) \\
\psi_{-n}(x, y, \sigma)=\sum_{\mu} \rho_{\sigma, \mu} \psi_{+n}\left(L_{x}-x+1, y, \mu\right)
\end{gathered}
$$

As $z \rightarrow-\infty$, far from the disordered region, we impose the boundary condition that the allowed states consist exclusively of incoming and outgoing propagating waves. Thus in $z<0$ states with $\operatorname{Im} k_{n}>0$ are excluded. We denote by $2 N$ the number of "open channels", i.e. states with $\operatorname{Im} k_{n}=0$ that carry a positive probability current in the $+z$ direction; there are an equal number carrying current in the $-z$ direction. We label these states so that $+n$ carries a current in the $+z$ direction and $-n$ a current in the $-z$ direction. After a suitable normalisation of the transverse wavefunctions (see Appendix A) the electric current due to electrons at the left of the disordered section is

$$
I_{e}=\frac{-e}{h} \sum_{n ; \operatorname{Im} k_{n}=0}\left|a_{+n}\right|^{2}-\left|a_{-n}\right|^{2}
$$

The boundary condition as $z \rightarrow-\infty$, imposed above, ensures that only open channels, and not "closed channels" with $\operatorname{Im} k_{n} \neq 0$, contribute to the current. A similar expansion may be made on the right in terms of a set of coefficients $\left\{a_{+n}^{\prime}, a_{-n}^{\prime}\right\}$ with the boundary condition that we admit only those states with $\operatorname{Im} k_{n} \geq 0$. Thus, far to the right of the 
disordered section, as $z \rightarrow+\infty$, the allowed states again consist exclusively of incoming and outgoing propagating waves.

The $4 N \times 4 N$ scattering matrix for electrons $S_{e}$ relates the $4 N$ incoming flux amplitudes at the left, $a_{+}=$ $\left\{a_{+n} ; \operatorname{Im} k_{n}=0\right\}$ and the right $a_{-}^{\prime}=\left\{a_{-n}^{\prime} ; \operatorname{Im} k_{n}=0\right\}$ with the $4 N$ outgoing flux amplitudes at the left $a_{-}=\left\{a_{-n} ; \operatorname{Im} k_{n}=0\right\}$ and the right $a_{+}^{\prime}=\left\{a_{+n}^{\prime} ; \operatorname{Im} k_{n}=0\right\}$

$$
S_{e}\left[\begin{array}{l}
a_{+} \\
a_{-}^{\prime}
\end{array}\right]=\left[\begin{array}{l}
a_{-} \\
a_{+}^{\prime}
\end{array}\right]
$$

The matrix $S_{e}$ has the structure

$$
S_{e}=\left[\begin{array}{ll}
r_{e} & t_{e}^{\prime} \\
t_{e} & r_{e}^{\prime}
\end{array}\right]
$$

in terms of the $2 N \times 2 N$ reflection and transmission matrices for left incidence $\left(r_{e}, t_{e}\right)$ and right incidence $\left(r_{e}^{\prime}, t_{e}^{\prime}\right)$.

Since we are considering time independent scattering, the currents to the left and right of the disordered section must be equal, and therefore it follows that $S_{e}$ is unitary. There is an additional restriction on $S_{e}$ when, in the absence of an applied magnetic field, the Hamiltonian is time reversal invariant ie. $\left[H_{e}, \mathcal{T}\right]=0$ with $\mathcal{T}$ given in Eq. $(2.5)$. For a suitable choice of transverse wavefunctions (see Appendix A) $S_{E}$ will then satisfy

$$
S_{e}^{T}=\left[\begin{array}{cc}
1_{2 N} & 0 \\
0 & -1_{2 N}
\end{array}\right] S_{e}\left[\begin{array}{cc}
-1_{2 N} & 0 \\
0 & 1_{2 N}
\end{array}\right]
$$

where $1_{2 N}$ means the $2 N \times 2 N$ unit matrix. This can be written in the equivalent form

$$
\begin{aligned}
& r_{e}=-r_{e}^{T} \\
& r_{e}^{\prime}=-\left(r_{e}^{\prime}\right)^{T} \\
& t_{e}=+\left(t_{e}^{\prime}\right)^{T}
\end{aligned}
$$

The simplicity of these relations, compared with for example those of Ref [16], is related to the presence of $\rho$ in Eq. (3.5) (see Appendices A and B)

We now turn to the scattering matrix $S_{h}$ for the holes. From the BdG equations we can see that the hole wavefunction $\psi^{h}$ evolves according to

$$
H_{h} \psi^{h}=i \hbar \frac{\partial}{\partial t} \psi^{h}
$$

where $H_{h}$ is given by

$$
H_{h}[+B]=\mathcal{T} H_{e}[+B] \mathcal{T}=-H_{e}[-B]
$$

If $\psi^{h}$ describes the scattering of a hole with excitation energy $+\epsilon$ in a field $+B$ then $\mathcal{T} \psi^{h}$ describes the scattering of an electron at energy $-\epsilon$ also in field $+B$. We shall make use of this in two ways.

Firstly, outside the disordered region $B=0$ and $H_{h}=-H_{e}$. Thus outside the disordered region it is useful to expand $\psi^{h}$ in terms of the Bloch states of $H_{e}$ at energy $E_{F}-\epsilon$. At the left, for example

$$
\psi^{h}(x, y, z, \sigma)=\sum_{n ; \operatorname{Im} k_{n} \leq 0} b_{-n} \psi_{+n}(x, y, \sigma) \exp \left(+i k_{n} z\right)+\sum_{n ; \operatorname{Im} k_{n}=0} b_{+n} \psi_{-n}(x, y, \sigma) \exp \left(-i k_{n} z\right)
$$

Note that since in this region $H_{h}=-H_{e}$ the probability currents are reversed by comparison with the electron case. We therefore associate the coefficient $b_{+n}$, the flux amplitude for a positive hole probability current in the $+z$ direction, with the wavefunction proportional to $\exp \left(-i k_{n} z\right)$. The holes carry an opposite electric charge to that of the electrons so that they carry an electric current

$$
I_{h}=\frac{e}{h} \sum_{n ; \operatorname{Im} k_{n}=0}\left|b_{+n}\right|^{2}-\left|b_{-n}\right|^{2}
$$

By definition, the $4 N \times 4 N$ matrix $S_{h}$ relates incoming hole probability currents to outgoing probability currents

$$
S_{h}\left[\begin{array}{l}
b_{+} \\
b_{-}^{\prime}
\end{array}\right]=\left[\begin{array}{l}
b_{-} \\
b_{+}^{\prime}
\end{array}\right]
$$


The matrix $S_{h}$ has a structure similar to that of Eq. (3.8); i.e.

$$
S_{h}=\left[\begin{array}{ll}
r_{h} & t_{h}^{\prime} \\
t_{h} & r_{h}^{\prime}
\end{array}\right]
$$

Secondly by rewriting $\mathcal{T} \psi^{h}$ in terms of electron flux amplitudes, and recalling that these amplitudes are related by $S_{e}$, we arrive at a relation between $S_{e}(-\epsilon,+B)$ and $S_{h}(+\epsilon,+B)$

$$
S_{h}(+\epsilon,+B)=\left[\begin{array}{cc}
1_{2 N} & 0 \\
0 & -1_{2 N}
\end{array}\right] S_{e}^{*}(-\epsilon,+B)\left[\begin{array}{cc}
-1_{2 N} & 0 \\
0 & 1_{2 N}
\end{array}\right]
$$

or

$$
\begin{aligned}
& r_{h}(+\epsilon,+B)=-\left[r_{e}(-\epsilon,+B)\right]^{*} \\
& r_{h}^{\prime}(+\epsilon,+B)=-\left[r_{e}^{\prime}(-\epsilon,+B)\right]^{*} \\
& t_{h}(+\epsilon,+B)=+\left[t_{e}(-\epsilon,+B)\right]^{*} \\
& t_{h}^{\prime}(+\epsilon,+B)=+\left[t_{e}^{\prime}(-\epsilon,+B)\right]^{*}
\end{aligned}
$$

Again we assume here a suitable choice of transverse wavefunctions (see Appendix A.) Following a similar line of argument it is possible to demonstrate a relationship between $S_{e}(+\epsilon,+B)$ and $S_{e}(+\epsilon,-B)$.

\section{B. Andreev scattering at the NS interface}

In this section we outline the calculation of the coefficients of Andreev reflection [17] at the NS interface. These are essentially unchanged by the introduction of a spin orbit interaction in the materials forming the junction. We assume throughout that $\Delta_{0} \ll E_{F}$, a condition which is realised in practice.

Far from the NS interface in the normal metal the superconducting gap $\Delta \rightarrow 0$. On the other hand, far from the NS interface in the superconductor, $\Delta \rightarrow \Delta_{0} \exp (i \varphi)$ where $\Delta_{0}$ is real. In general the reflection coefficients will depend on the precise form of $\Delta$ in the transition region near the junction. For a point contact junction, however, it is permissible to assume a simple step model

$$
\begin{array}{ll}
\Delta=0 & z \notin \mathrm{S} \\
\Delta=\Delta_{0} \exp (i \varphi) & z \in \mathrm{S}
\end{array}
$$

We are interested in the situation where the energy $E=E_{F}+\epsilon$ of the incident electron is in the energy gap of the superconductor $E_{F}<E<E_{F}+\Delta_{0}$. Anticipating somewhat in order to avoid unnecessary algebra, we find the electron is mainly reflected as a hole like excitation. A solution of the BdG equation in the normal metal corresponding to this is

$$
\left[\begin{array}{c}
\psi^{e} \\
\psi^{h}
\end{array}\right]=\left[\begin{array}{l}
1 \\
0
\end{array}\right] \exp \left(i k_{n}^{(+)} z\right) \psi_{+n}^{(+)}(x, y, \sigma)+\left[\begin{array}{c}
0 \\
r_{h e}^{A}
\end{array}\right] \exp \left(i k_{n}^{(-)} z\right) \psi_{+n}^{(-)}(x, y, \sigma)
$$

where $r_{h e}^{A}$ denotes the matrix of Andreev reflection amplitudes and the superscript $( \pm)$ refer to Bloch states with energies $E^{( \pm)}=E_{F} \pm \epsilon$. The first term describes an excitation where an electron above the Fermi level is incident from the left in channel $n$. The second term corresponds to an excitation in which an electron below the Fermi level is annihilated, i.e. to a reflected "hole" with opposite velocity and spin to that of the incoming electron. Since $\Delta_{0} \ll E_{F}$ we can to a good approximation ignore the difference between $k_{n}^{(+)}$and $k_{n}^{(-)}$and similarly the differences between the transverse wavefunctions. Requiring that the wavefunction and its derivative be continuous at the boundary of the superconductor leads to

$$
r_{h e}^{A}=i \exp (-i \varphi), \quad r_{e h}^{A}=i \exp (+i \varphi)
$$

Here we have made the further assumption that $\epsilon \ll \Delta_{0}$, the limit of interest in what follows, and we have also given the reflection coefficient for an incident hole. 


\section{The Scottky barrier at the NS interface}

In real NS junctions, a mismatch between the conduction bands of the two materials which make up the junction results in the creation of a Schottky barrier at the interface. This barrier plays an important role in the physics of the device and so we must take it into account. We shall model the Schottky barrier as a simple planar potential barrier. At the barrier an incident particle may be either transmitted without a change of momentum or specularly reflected. We neglect any dependence of the reflection and transmission probabilities on the momentum of the incident particle so that the properties of the barrier are described by a single parameter $\Gamma \in[0,1]$, its transparency. The transmission and reflection matrices which make up the electron scattering matrix $S_{e}^{B}$ of the barrier are

$$
\begin{aligned}
& t_{e}^{B}=\sqrt{\Gamma} \quad 1_{2 N} \\
& t_{e}^{\prime B}=\sqrt{\Gamma} 1_{2 N} \\
& r_{e}^{B}=\sqrt{1-\Gamma} w_{B} \\
& r_{e}^{\prime B}=-\sqrt{1-\Gamma} w_{B}^{\dagger}
\end{aligned}
$$

The precise form of the $2 N \times 2 N$ matrix $w_{B}$ depends on the choice of transverse wavefunctions. For subsequent analysis we need only note, however, that $w_{B}$ is antisymmetric and unitary. The hole scattering matrix $S_{h}^{B}$ for the barrier is related to $S_{e}^{B}$ in the usual way by (3.18).

\section{Combination of scattering matrices}

It is the purpose of this section, having considered above the scattering matrices for the various components of the NS junction, to explain how the scattering matrices may be combined to find the total scattering matrix. We consider first a junction without a Schottky barrier. For the normal part we can write

$$
\left[\begin{array}{ll}
r & t^{\prime} \\
t & r^{\prime}
\end{array}\right]\left[\begin{array}{l}
c_{+} \\
c_{-}^{\prime}
\end{array}\right]=\left[\begin{array}{l}
c_{-} \\
c_{+}^{\prime}
\end{array}\right]
$$

where

$$
r=\left[\begin{array}{cc}
r_{e} & 0 \\
0 & r_{h}
\end{array}\right] \text { etc. }
$$

are $4 N$-dimensional matrices and

$$
c_{ \pm}=\left[\begin{array}{l}
a_{ \pm} \\
b_{ \pm}
\end{array}\right], c_{ \pm}^{\prime}=\left[\begin{array}{c}
a_{ \pm}^{\prime} \\
b_{ \pm}^{\prime}
\end{array}\right]
$$

$4 N$-dimensional vectors, in the notation of Sec. IV. For the Andreev part we define the $4 N \times 4 N$ scattering matrix $S^{A}$ by

$$
S^{A} c_{+}^{\prime}=c_{-}^{\prime}
$$

By reference to Section III. this has the form

$$
S^{A}=\left[\begin{array}{cc}
0 & r_{e h}^{A} 1_{2 N} \\
r_{h e}^{A} 1_{2 N} & 0
\end{array}\right]
$$

For the combined system, the $4 N \times 4 N S$ matrix is defined by

$$
S c_{+}=c_{-}
$$

In equation (3.23) we replace $c_{-}^{\prime}$ in terms of $c_{+}^{\prime}$ as in (3.26); we then perform the matrix multiplication and, from the two resulting equations, eliminate $c_{+}^{\prime}$. From the expression relating $c_{+}$and $c_{-}$we extract the $4 N \times 4 N S$ matrix of (3.28) to obtain

$$
S=r+t^{\prime} S^{A} \frac{1}{1-r^{\prime} S^{A}} t
$$


In the electron-hole spaces, $S$ of Eq. (3.29) has the structure

$$
S=\left[\begin{array}{ll}
r_{e e} & r_{e h} \\
r_{h e} & r_{h h}
\end{array}\right]
$$

$r_{e e}$ etc. being $2 N \times 2 N$ matrices. To determine the conductance we shall need the submatrix $r_{h e}$. From Eqs. (3.29) and (3.30) we find

$$
r_{h e}=t_{h}^{\prime}\left(S^{A} \frac{1}{1-r^{\prime} S^{A}}\right)_{h e} t_{e}
$$

Using the structure (3.27) of $S^{A}$ we can write $r_{h e}$ as

$$
r_{h e}=t_{h}^{\prime} r_{h e}^{A}\left(\frac{1}{1-r^{\prime} S^{A}}\right)_{e e} t_{e}
$$

For any nonsingular operator $D$ we now use the operator identity [18]

$$
\left(\frac{1}{D}\right)_{e e}=\left(\frac{1}{D_{e e}-D_{e h} \frac{1}{D_{h h}} D_{h e}}\right)_{e e}
$$

to find

$$
r_{h e}=t_{h}^{\prime} r_{h e}^{A}\left[1-r_{e}^{\prime} r_{h e}^{A} r_{h}^{\prime} r_{e h}^{A}\right]^{-1} t_{e}
$$

For a junction with a Schottky barrier we first consider the composition of the Schottky barrier and the superconductor. The scattering matrix for this system can be obtained from Eq. (3.29) where $r, t$ and $t^{\prime}$ are taken from the model (3.22) for the barrier. With the aid of the identity (3.33) and the following one [18]

$$
\left(\frac{1}{D}\right)_{h e}=-\frac{1}{D_{h h}} D_{h e}\left(\frac{1}{D_{e e}-D_{e h} \frac{1}{D_{h h}} D_{h e}}\right)_{e e}
$$

we find

$$
\begin{gathered}
S^{B S}=\left[\begin{array}{cc}
r_{e e}^{B S} & r_{e h}^{B S} \\
r_{h e}^{B S} & r_{h h}^{B S}
\end{array}\right] \\
r_{h e}^{B S}=i \exp (-i \varphi)[\Gamma /(2-\Gamma)] 1_{2 N} \\
r_{e h}^{B S}=-\left(r_{h e}^{[B S]}\right)^{*} \\
r_{e e}^{B S}=2[\sqrt{1-\Gamma} /(2-\Gamma)] w_{B} \\
r_{h h}^{B S}=-\left(r_{e e}^{[B S]}\right)^{*}
\end{gathered}
$$

The scattering matrix for the complete system of normal part, Schottky barrier and superconductor can now be obtained from Eq. (3.29), where $r^{\prime}$, etc. refer to the normal metal as before, but $S^{A}$ is replaced by $S^{B S}$ of (3.37). (Note that in deriving Eq. (3.29) the structure (3.27) of $S^{A}$ was not used.)

\section{CONDUCTANCE FORMULAE}

We assume that the bias voltage is small in comparison to $\Delta_{0} / e$, and that the size of the superconducting part is long enough so that there is no quasi-particle current in the superconductor. In this case, the zero temperature dc conductance $G_{N S}$ of the normal-superconducting junction can be described by a simplified "Landauer" formula which has been derived in Ref. [19-21]

$$
G_{N S}=\frac{2 e^{2}}{h} g_{N S}=\frac{2 e^{2}}{h} \operatorname{tr} r_{h e} r_{h e}^{\dagger}
$$

where $r_{h e}$ is the $2 N \times 2 N$ matrix of electron-hole reflection amplitudes for the composite system. 
There is an important simplification if the Hamiltonian of the system is time reversal invariant and the bias voltage is small in comparison to the Thouless energy $E_{c}[22]$, so that the energy dependence of $S_{e}$ can be neglected. The conductance then has the form [23]

$$
G_{N S}=\frac{2 e^{2}}{h} \sum_{n=1}^{2 N}\left[\frac{T_{n}}{2-T_{n}}\right]^{2}
$$

where $T_{n}$ are the eigenvalues of $t_{e} t_{e}^{\dagger}$. We have verified that this result still holds when spin rotation symmetry is broken by the spin orbit interaction.

In what follows we wish to compare the quantum conductance of the NS junction, calculated from (4.1), with the classical conductance of the junction. This latter quantity is determined using the classical rule of combining conductances

$$
1 / g=1 / g_{1}+1 / g_{2}
$$

This corresponds to the addition of flux intensities as opposed to flux amplitudes. The conductance associated with the electron traversing the normal part is $2 \mathrm{~N} / \mathrm{s}$ and similarly for the hole in the traversing the normal part in the opposite direction. The conductance of the barrier is $2 N\left|r_{h e}^{B S}\right|^{2}$, so that the classical conductance is

$$
g_{N S}^{c l}=\frac{2 N\left|r_{h e}^{B S}\right|^{2}}{1+2 s\left|r_{h e}^{B S}\right|^{2}}
$$

The classical conductance is insensitive to the breaking of time reversal and spin rotation symmetries.

\section{QUASI-BALLISTIC JUNCTION}

In this section we shall calculate the conductance of an NS junction to first order in $s=L / l$, where $L$ is the length of the normal part of the junction and $l$ is the elastic mean free path. We neglect terms of order $s^{2}$ and above so that result is strictly applicable only in the limit that $s \ll 1$. Nevertheless we shall see that the results of the calculation shed considerable light on the origin of the quantum interference in the device.

The $2 N \times 2 N$ reflection matrix $r_{h e}$ for the system consisting of the normal metal, barrier and superconductor can be obtained as discussed in Section III D. After expanding to second order in $r_{e}^{\prime}, r_{h}^{\prime}$, which is sufficient for an evaluation of $g_{N S}$ to first order in $s$, we have

$$
\begin{aligned}
r_{h e} & =t_{h}^{\prime} r_{h e}^{B S} t_{e}+t_{h}^{\prime} r_{h e}^{B S} r_{e}^{\prime} r_{e e}^{B S} t_{e}+t_{h}^{\prime} r_{h h}^{B S} r_{h}^{\prime} r_{h e}^{B S} t_{e}+t_{h}^{\prime} r_{h e}^{B S} r_{e}^{\prime} r_{e e}^{B S} r_{e}^{\prime} r_{e e}^{B S} t_{e} \\
& +t_{h}^{\prime} r_{h h}^{B S} r_{h}^{\prime} r_{h e}^{B S} r_{e}^{\prime} r_{e e}^{B S} t_{e}+t_{h}^{\prime} r_{h h}^{B S} r_{h}^{\prime} r_{h h}^{B S} r_{h}^{\prime} r_{h e}^{B S} t_{e}+t_{h}^{\prime} r_{h e}^{B S} r_{e}^{\prime} r_{e h}^{B S} r_{h}^{\prime} r_{h e}^{B S} t_{e}+\ldots
\end{aligned}
$$

The conductance is found by substituting this into (4.1) and performing an average over an ensemble of scattering matrices $S_{e}$, describing a set of microscopically different but macroscopically equivalent configurations of impurities in the normal part of the junction.

In principle, the distribution for $S_{e}$ should be calculated from some model distribution of Hamiltonians. We shall not, however, attempt to do that here. Instead we will assume that the resulting ensemble of scattering matrices $S_{e}$ is distributed according to the "local maximum entropy model" [3,24,25]. If the geometry is quasi- $1 d$ and the number of channels $N$ sufficiently large, then results obtained with the aid of the local maximum entropy model are known to be identical to those obtained from the class of microscopic models described by the the nonlinear sigma model $[26,27]$.

The distribution of $S_{e}$ in the local model depends on $N, s$ and the symmetry of the Hamiltonian, i.e. whether or not time reversal symmetry is broken and whether or not spin rotation symmetry is broken. There are four ensembles (Table. I.) The critical strengths of the magnetic field and the spin orbit interaction separating the various ensembles should be similar to those associated with the weak localisation effect in normal metals. The details of the distribution of $S_{e}$ for the four ensembles can be found in Appendix C.

It will be helpful, when we come to discuss the interpretation of the results, to write (5.1) in the form

$$
r_{h e}=\sum_{i=1}^{\infty} p_{i}
$$

Each term in the series represents the contribution of a particular scattering processes to $r_{h e}$. The classical conductance is obtained by ignoring interference between different processes and summing intensities 


$$
g_{N S}^{c l}=\operatorname{tr}\left\langle\sum_{i=1}^{\infty} p_{i} p_{i}^{\dagger}\right\rangle
$$

The quantum correction to this classical conductance is found by summing the interference between different processes

$$
\delta g_{N S}=\operatorname{tr}\left\langle\sum_{i \neq j} p_{i} p_{j}^{\dagger}\right\rangle
$$

After carrying out the average we find that

$$
g_{N S}^{c l}=2 N\left|r_{h e}^{B S}\right|^{2}\left(1-2\left|r_{h e}^{B S}\right|^{2} s+O\left(s^{2}\right)\right)
$$

which agrees with the expansion of (4.4) to the order we are considering. The explicit expressions for $\delta g_{N S}$ are collected together in Table II. The function $f$ of the barrier transparency, which appears in the table, has the explicit form:

$$
f(\Gamma)=\frac{2 \Gamma^{2}}{(2-\Gamma)^{2}}\left[\frac{2 \Gamma^{2}}{(2-\Gamma)^{2}}-1\right]
$$

There are two obvious limiting cases: $\Gamma=1$ corresponding to a junction without a Schottky barrier and $\Gamma \ll 1$ corresponding to a junction with a high Schottky barrier. The first point to note is that the quantum correction is of $O(N)$, the same order as the classical conductance, in zero field. Secondly for $\Gamma \ll 1$, the conductance increases as disorder is added to the junction. This is the essence of the dramatic reflectionless tunnelling effect which we discuss in Section VIII. Thirdly when time reversal symmetry is broken by the application of a magnetic field the quantum correction is $O\left(N^{0}\right)$ and not $O(1 / N)$ as might have been expected by analogy with the weak localisation effect in a normal metal. The final, and perhaps the most surprising result, is that in a magnetic field the breaking of spin rotation symmetry by the spin orbit interaction multiplies the quantum correction by a factor of minus one half even though the symmetry of the Hamiltonian, in the sense of random matrix theory, is unchanged and remains unitary.

\section{SEMICLASSICAL INTERPRETATION}

The importance of quantum interference between processes in which the path of an electron (hole) incident on the NS boundary is subsequently retraced by an Andreev reflected hole (electron) was first pointed out in [5]. In the absence of a magnetic field, and if the bias voltage is small enough, electrons which move along a path in one given sense are phase conjugated with holes traversing the time reversed path. In a magnetic field, or if the bias voltage is large enough, this phase conjugation is destroyed. However we have seen that there is a significant quantum correction even in a magnetic field. There must therefore be an additional source of quantum interference which is not sensitive to the breaking of time reversal symmetry. As we shall see the relevant processes involve paths in which an electron (hole) and an Andreev reflected hole (electron) traverse a loop in the same sense. In order to remain concise, we shall refer to such processes as containing identical paths. The interference involving such paths can only be destroyed by applying a large enough bias voltage. The physical importance of the bias voltage as a "symmetry breaking parameter" is discussed in [10].

Only some of the terms in (5.4) are found to be nonzero after averaging, so that in fact

$$
\delta g_{N S}=\operatorname{tr}\left\langle p_{1} p_{5}^{\dagger}+p_{1} p_{7}^{\dagger}+p_{5} p_{1}^{\dagger}+p_{7} p_{1}^{\dagger}\right\rangle+O\left(s^{2}\right)
$$

where $p_{j}$ means the $j$ th term in (5.1.) Consider first the interference between process $p_{1}$ and $p_{5}$. An example of a scattering path which contributes to process $p_{1}$

$$
p_{1}=t_{h}^{\prime} r_{h e}^{B S} t_{e}
$$

is illustrated in Figure 2. Since we are working to first order in $s$ it is sufficient to consider the motion of the electron and holes, traversing from one side of the normal part of the junction to the other, as ballistic, so these trajectories appear as straight lines in Figure 2. Examples of paths which contribute to $p_{5}$

$$
p_{5}=t_{h}^{\prime} r_{h h}^{B S} r_{h}^{\prime} r_{h e}^{B S} r_{e}^{\prime} r_{e e}^{B S} t_{e}
$$

are illustrated in Figures 3 and 4 . To first order in $s$ the interference between processes $p_{1}$ and $p_{5}$ is 


$$
\operatorname{tr}\left\langle p_{1} p_{5}^{\dagger}+p_{5} p_{1}^{\dagger}\right\rangle=2\left|r_{h e}^{B S}\right|^{2}\left|r_{e e}^{B S}\right|^{2} \operatorname{tr}\left\langle r_{h}^{\prime} r_{e}^{\prime}\right\rangle
$$

The remaining terms involving $p_{1}$ and $p_{7}$ contribute

$$
\operatorname{tr}\left\langle p_{1} p_{7}^{\dagger}+p_{7} p_{1}^{\dagger}\right\rangle=-2\left|r_{h e}^{B S}\right|^{4} \operatorname{tr}\left\langle r_{h}^{\prime} r_{e}^{\prime}\right\rangle
$$

In the interest of brevity we will concentrate on the interference between $p_{1}$ and $p_{5}$. A very similar analysis is possible for the interference between processes $p_{1}$ and $p_{7}$ leading to identical conclusions. We proceed by relating the product of electron and hole reflection matrices in (6.4) to a product of an electron and a hole Greens function. To simplify the algebra we shall suppose that both the spin orbit interaction and the magnetic field are zero everywhere except in the disordered region. We shall also suppose that $L_{y}=1$ and impose periodic boundary conditions in the $x$ direction. The Bloch states at energy $E$ have the form

$$
\begin{array}{llll}
\psi_{2 m}(x, z, \sigma) & =\exp \left(i k_{2 m}^{x} x\right) \exp \left(i k_{2 m} z\right) \delta_{\sigma, \sigma_{2 m}} & \sigma_{2 m} & =\uparrow \\
\psi_{2 m+1}(x, z, \sigma) & =\exp \left(i k_{2 m+1}^{x} x\right) \exp \left(i k_{2 m+1} z\right) \delta_{\sigma, \sigma_{2 m+1}} & \sigma_{2 m+1} & =\downarrow \\
\psi_{-2 m}(x, z, \sigma) & =-\exp \left(-i k_{2 m}^{x} x\right) \exp \left(-i k_{2 m} z\right) \delta_{\sigma, \sigma_{-2 m}} & \sigma_{-2 m} & =\downarrow \\
\psi_{-(2 m+1)}(x, z, \sigma) & =\exp \left(-i k_{2 m+1}^{x} x\right) \exp \left(-i k_{2 m+1} z\right) \delta_{\sigma, \sigma_{-(2 m+1)}} & \sigma_{-(2 m+1)} & =\uparrow
\end{array}
$$

where

$$
k_{2 m}^{x}=k_{2 m+1}^{x}=\frac{2 \pi}{L_{x}} m=0, \ldots, L_{x}-1
$$

and the energy and the momenta are related by

$$
E=2 \cos k_{m}^{x}+2 \cos k_{m}
$$

The reflection matrices for electrons and holes can be related to the corresponding Green's functions as indicated in Appendix A.

$$
\begin{aligned}
{\left[r_{e}^{\prime}\right]_{m n}=} & -i \sqrt{4 \sin k_{m} \sin k_{n}} \exp \left(+i\left(k_{m}+k_{n}\right) L\right) \\
& \sum_{x_{a} \sigma x_{b} \sigma^{\prime}} \psi_{+m}^{*}\left(x_{b} \sigma\right) G_{e}^{+}\left(x_{b}, L, \sigma ; x_{a}, L, \sigma^{\prime}\right) \psi_{-n}\left(x_{a} \sigma^{\prime}\right) \\
{\left[r_{h}^{\prime}\right]_{n m}=} & -i \sqrt{4 \sin k_{m} \sin k_{n}} \exp \left(-i\left(k_{m}+k_{n}\right) L\right) \\
& \sum_{x_{a} \sigma x_{b} \sigma^{\prime}} \psi_{-n}^{*}\left(x_{b} \sigma\right) G_{h}^{+}\left(x_{b}, L, \sigma ; x_{a}, L, \sigma^{\prime}\right) \psi_{+m}\left(x_{a} \sigma^{\prime}\right)
\end{aligned}
$$

Note that, for convenience, the states (6.6) have not been normalised to carry identical currents, proper account of this has been taken in the expressions (6.9) and (6.10) for the reflection matrices.

The quantum correction involves a trace over the product of $r_{e}$ and $r_{h}$. Using the relation with the Green's function this can be separated into two elements: an integration over the cross section involving the transverse wavefunctions and an average of the product of an electron and a hole Greens function. We will consider the second element first. This involves evaluating

$$
\Lambda_{\sigma_{-n}, \sigma_{+m}}\left(x_{a}, x_{b}\right)=\left\langle G_{h}^{+}\left(x_{a}, L, \sigma_{-n} ; x_{b}, L, \sigma_{+m}\right) G_{e}^{+}\left(x_{b}, L, \sigma_{+m} ; x_{a}, L, \sigma_{-n}\right)\right\rangle
$$

Within the semiclassical approximation, as is explained in Ref. [28], Ch. 12 and Ch. 13, we can express the Greens functions as summations over paths:

$$
\begin{aligned}
& G_{e}^{+}\left(x_{b}, L, \sigma_{+m} ; x_{a}, L, \sigma_{-n}\right)=\sum_{j: x_{a} \rightarrow x_{b}} A_{j} \exp \left(i S_{j}+i \Phi_{j}\right)\left[v_{j}\right]_{\sigma_{+m}, \sigma_{-n}} \\
& G_{h}^{+}\left(x_{b}, L, \sigma_{-n} ; x_{a}, L, \sigma_{+m}\right)=-\sum_{j: x_{a} \rightarrow x_{b}} A_{j} \exp \left(-i S_{j}-i \Phi_{j}\right)\left[v_{j}\right]_{\sigma_{-n}, \sigma_{+m}}
\end{aligned}
$$

Substituting (6.12) into (6.11) and taking the disorder average we find that only two contributions remain: 


\section{A. Time reversed paths}

The path of the electron moving between $x_{a}$ and $x_{b}$ is retraced by the Andreev reflected hole as illustrated in Figure 3. The electron and hole charges are opposite but they traverse the path in opposite directions so the Aharonov-Bohm phase factors they accumulate do not cancel each other out. Thus this term is important only when the magnetic field is negligible and the Hamiltonian is time reversal symmetric.

Let us consider first the situation where the spin orbit interaction is negligible. In the quasi- $1 d$ limit, the average of the product of the hole and electron Green's functions is independent of $x_{a}$ and $x_{b}$ so that

$$
\Lambda_{\sigma_{-n}, \sigma_{+m}}\left(x_{a}, x_{b}\right)=-\lambda \delta_{\sigma_{-n}, \sigma_{+m}}
$$

After integrating the product of transverse wavefunctions over the cross section we find

$$
\left\langle\left[r_{h}^{\prime}\right]_{m n}\left[r_{e}^{\prime}\right]_{n m}\right\rangle=4 \sin k_{m} \sin k_{n} \lambda \delta_{\sigma_{-n}, \sigma_{+m}}+\text { I.P. }
$$

where I.P. denotes the contribution due to identical paths which we will consider below. We determine $\lambda$ by demanding consistency with the local maximum entropy model. In this model $S_{e}$ is distributed isotropically (see Appendix C) so that averages, such as that in (6.14), are independent of the channel indices $n$ and $m$. The extent to which the isotropy assumption is reasonable for microscopic models is discussed in Refs. [29,30]. In the present case, to maintain consistency with the results of Section $\mathrm{V}$, we are required to make the approximation that the group velocities appearing in (6.14) can be replaced by an average velocity. This is a reasonable assumption if the energy is such that we are far from any subband edges, but is of questionable validity for energies close to a subband edge. Having made this approximation and determined $\lambda$ by comparison with Section $V$ we find

$$
<\left(r_{h}^{\prime}\right)_{2 n+1,2 m}\left(r_{e}^{\prime}\right)_{2 m, 2 n+1}>=<\left(r_{h}^{\prime}\right)_{2 n, 2 m+1}\left(r_{e}^{\prime}\right)_{2 m+1,2 n}>=\frac{s}{N+1}
$$

If the spin orbit interaction is appreciable we assume that the spin direction of an electron or hole traversing a path is completely randomised. The matrix $v$ describing this rotation can then be assumed to be uniformly distributed, relative to the invariant measure, on the group $S U(2)$. The result is

$$
<\left(r_{h}^{\prime}\right)_{n, m}\left(r_{e}^{\prime}\right)_{m, n}>=\frac{s}{2 N-1}
$$

After summation over all channels the contribution of this term to the quantum correction is essentially independent of the strength of the spin orbit interaction. This is because the spin rotation experienced by an electron traversing a path in a given sense cancels that of the hole traversing the same path in the opposite sense. To demonstrate this explicitly it suffices to note that after summation over spin indices the contribution of time reversed paths is proportional to $\operatorname{tr} v^{\dagger} v=\operatorname{tr} 1_{2}$, which is a constant independent of the distribution of $v$.

\section{B. Identical paths $x_{a}=x_{b}$}

The electron path in the normal metal contains a loop which is retraced in the same sense by the Andreev reflected hole as illustrated in Figure 4. The Aharonov-Bohm phase accumulated by the electron and hole, are now exactly opposite and cancel. Therefore this term is important even if time reversal symmetry is broken.

Proceeding in a similar way to that above we find that the contribution of identical paths is

$$
\left\langle\left[r_{h}^{\prime}\right]_{n m}\left[r_{e}^{\prime}\right]_{m n}\right\rangle=4 \sin k_{m} \sin k_{n} \lambda \delta_{m, n}\left\langle v_{\sigma_{-n}, \sigma_{+m}} v_{\sigma_{+m}, \sigma_{-n}}\right\rangle+\text { T.R.P. }
$$

(Here T.R.P. refers to the contribution from time reversed paths.) The presence of the delta function, arising from the integration of the transverse functions over the cross section, means that identical paths only contribute for electrons and holes which are back scattered by the disordered region as is indicated in Figure 4. Note the novel feature that this is true even if time reversal symmetry is broken in the disordered region. The average over the matrices $v$ is calculated with respect to the distribution $p(v)=\delta\left(v-1_{2}\right)$ if the spin orbit interaction is negligible, and $p(v)$ uniform on $S U(2)$, if it is appreciable. As above we determine $\lambda$ by reference to Section V. If the spin orbit interaction is negligible we find:

$$
<\left(r_{h}^{\prime}\right)_{2 n+1,2 m}\left(r_{e}^{\prime}\right)_{2 m, 2 n+1}>=<\left(r_{h}^{\prime}\right)_{2 n, 2 m+1}\left(r_{e}^{\prime}\right)_{2 m+1,2 n}>=\delta_{m, n} \frac{s}{N+1}
$$


in zero magnetic field and:

$$
<\left(r_{h}^{\prime}\right)_{2 n+1,2 m}\left(r_{e}^{\prime}\right)_{2 m, 2 n+1}>=<\left(r_{h}^{\prime}\right)_{2 n, 2 m+1}\left(r_{e}^{\prime}\right)_{2 m+1,2 n}>=\delta_{m, n} \frac{s}{N}
$$

in a nonzero field. If the spin orbit interaction is appreciable we find

$$
<\left(r_{h}^{\prime}\right)_{n, m}\left(r_{e}^{\prime}\right)_{m, n}>=\delta_{m, n} \frac{-s}{2 N-1}
$$

in zero magnetic field and

$$
<\left(r_{h}^{\prime}\right)_{n, m}\left(r_{e}^{\prime}\right)_{m, n}>=\delta_{m, n} \frac{-s}{2 N}
$$

in a nonzero field. The change of sign when spin rotation symmetry is broken comes about because an electron and a hole traversing the same path in the same direction undergo the same spin rotation. After summation over the spin indices, the contribution from identical paths is thus proportional to $\operatorname{tr} v^{2}$ which is sensitive to the distribution of $v$

$$
\begin{aligned}
& \operatorname{tr} v^{2}=+2 p(v)=\delta\left(v-1_{2}\right) \\
& =-1 p(v) \text { uniform on } S U(2)
\end{aligned}
$$

\section{DIFFUSIVE JUNCTION WITHOUT A SCHOTTKY BARRIER $(\Gamma=1)$}

For convenience we will defer discussion of a junction for which the Scottky barrier has a low transparency $(\Gamma \ll 1)$ and which exhibit reflectionless tunnelling, to Section VIII, and focus in this section on junctions without a Schottky barrier.

We now have to sum the perturbation series (5.1) to all orders in $s$ to find the quantum correction for a diffusive junction. In the absence of a magnetic field, this amounts to the evaluation of the integral:

$$
g_{N S}=\int_{0}^{1} \mathrm{~d} T \rho(T)\left[\frac{T}{2-T}\right]^{2}
$$

Here $\rho(T)$ is the density of the eigenvalues of the matrix $t_{e} t_{e}^{\dagger}$, where $t_{e}$ refers to the composite system composed of the normal metal and Schottky barrier.

Within the local maximum entropy model, the development of the density $\rho(T)$ as a function of $s$ is described by a nonlinear diffusion equation [31]. The initial condition on $\rho(T)$ at $s=0$ is:

$$
\rho_{s=0}(T)=N \delta(T-1)
$$

In the diffusive regime the solution to this equation is independent of the ensemble up to corrections of $O\left(N^{0}\right)$ [32]. Evaluating (7.1) we find an identical $O(N)$ contribution to $g_{N S}$ for the Orthogonal and Symplectic Ensembles, while the following $O\left(N^{0}\right)$ term is ensemble dependent. For the details, we refer the reader to [32]. With the aid of this reference we obtain the results for the Orthogonal and Symplectic Ensembles given in Table III.

For a diffusive junction in a magnetic field the summation of the perturbation series requires the performance of a number of averages over the unitary group. The authors of Ref. [10] find for the Unitary I Ensemble and $\Gamma=1$ that

$$
g_{N S}=\frac{2 N}{1+2 s}-\frac{8 s^{3}+12 s^{2}+12 s}{3(1+2 s)^{3}}+O(1 / N)
$$

This gives the same result as Section $\mathrm{V}$, in the limit that $s \ll 1$ and yields $\delta g_{N S}=-1 / 3$ in the diffusive regime $\left(1 \ll s \ll N\right.$.) As for the quasi-ballistic junction, the quantum correction is $O\left(N^{0}\right)$, not $O(1 / N)$, in a magnetic field.

The analysis of Ref [10] can be extended to the Unitary II Ensemble. In the absence of a Schottky barrier we have from (3.34) and (3.18):

$$
r_{h e}=i \exp (-i \varphi) t^{*} \sum_{p=0}^{\infty}\left[-r_{e}^{\prime} r_{e}^{*}\right]^{p} t_{e}
$$

Then with the aid of $(\mathrm{C} 1)$ and (4.1) we obtain for the conductance: 


$$
g_{N S}=\operatorname{tr}\left[T \sum_{p=0}^{\infty}\left[-u \sqrt{R} u^{*} \sqrt{R}\right]^{p} u T u^{\dagger} \sum_{q=0}^{\infty}\left[-\sqrt{R} u^{T} \sqrt{R} u^{\dagger}\right]^{q}\right]
$$

where $u=u_{4}^{*} u_{2}$ is uniformly distributed on $U(2 N)$. Following Ref [10] the ensemble average is accomplished in two steps. First the average on the unitary group and then the average over the distribution of $T$. The final result for the Unitary II Ensemble and $\Gamma=1$ is:

$$
g_{N S}=\frac{2 N}{1+2 s}+\frac{4 s^{3}+6 s^{2}+6 s}{3(1+2 s)^{3}}+O(1 / N)
$$

which reproduces the result of Section $\mathrm{V}$ for $s \ll 1$ and yields $\delta g_{N S}=+1 / 6$ in the diffusive regime.

The most interesting result is that, like the quasi-ballistic junction, the quantum correction is sensitive to the spin orbit interaction even if time reversal symmetry is broken. Referring to Section VI, it seems reasonable to assume that it is interference involving identical paths which is responsible for this sensitivity.

In order to confirm that quantum correction is sensitive to the spin orbit interaction, even if the junction is a magnetic field we have a carried out a numerical simulation. As a model for the junction we use the Hamiltonian of Section III. The Fermi energy $E_{F}$ is measured from centre $E_{0}$ of the energy band of the model. Since the zero of energy is arbitrary we can set $E_{0}=0$ for convenience. In the normal part of the junction, $0 \leq z \leq L-1$, we take the diagonal Hamiltonian elements as:

$$
\begin{array}{ll}
<x, y, z, \sigma|H| x, y, z, \mu>=U(x, y, z) \delta_{\sigma, \mu} & 0 \leq z \leq L_{z}-1 \\
<x, y, z, \sigma|H| x, y, z, \mu>=U_{0} \delta_{\sigma, \mu} & z=L
\end{array}
$$

The potential $U(x, y, z)$ is the random potential due to impurities and $U_{0}$ the height of the Schottky barrier. For simplicity we shall assume a spatially uncorrelated potential with a distribution $P(U)=p(U) \mathrm{d} U$

$$
\begin{array}{ll}
p(U)=1 / W & -W / 2 \leq U \leq+W / 2 \\
p(U)=0, & \text { otherwise. }
\end{array}
$$

To the right, in $z \geq L+1$, is the superconductor. The conductance is calculated using the standard Green's function iteration technique to produce $S_{e}$ and then using the formulae of Sections III and IV.

In Figure 5 we present the data for the junction conductance versus the parameter $\theta$, defined in Section III, controlling the spin orbit interaction. The transverse dimensions of the junction are $L_{x}=L_{y}=5$ and the longitudinal dimension $L=25$. The Fermi energy $E_{F}=0$. For these parameters $N=17$. We are interested in the limit $\Gamma=1$ so we set $U_{0}=0$. A magnetic field corresponding to a flux of $1 / 25 \phi_{0}$ per lattice cell is applied perpendicular to the junction corresponding to five flux quanta through the device. The distribution of the random potential has width $W=3$ corresponding to an estimated mean free path $l \simeq 4.3$. The junction is in the diffusive $\left(L_{z} \gg l\right)$ quasi- $1 d$ $\left(L_{x}, L_{y} \leq l, L_{x}, L_{y} \ll L_{z}\right)$ regime. The spin rotations between lattice sites are not random but differ depending on the direction of the displacement. Since these rotations do not commute the spin direction of the electron is effectively randomised and so, for $\theta$ large enough, the Symplectic Ensemble should be appropriate.

As the magnitude of the spin orbit interaction is increased the junction conductance $G_{N S}$ increases, while the normal conductance $G_{N}$ remains constant. This is exactly the behaviour predicted. The value $\delta G_{N S} \simeq 0.8 e^{2} / h$ obtained in the simulation is also in reasonable agreement with theoretical value $\delta G_{N S}=e^{2} / h$.

\section{REFLECTIONLESS TUNNELLING}

This is an effect observed in diffusive junctions when $\Gamma \ll 1$. In the absence of a magnetic field we may calculate the conductance in the same way as for the diffusive junction with $\Gamma=1$ but with the initial condition:

$$
\rho_{s=0}(T)=N \delta(T-\Gamma)
$$

instead of (7.2). At $O(N)$ the result is the same for both the Orthogonal and Symplectic Ensembles:

$$
\begin{aligned}
& g_{N S}=N \Gamma^{2} / 2+N s \Gamma^{2} \quad 1 \ll s \ll N ; \Gamma s \ll 1 \\
& g_{N S}=N /(s+1 / \Gamma) \quad 1 \ll s \ll N ; \Gamma s \gg 1
\end{aligned}
$$


We expect ensemble dependent corrections to this result at $O\left(N^{0}\right)$, though we have not calculated them explicitly. This quantum conductance has to be compared to the classical conductance obtained from Eq. (4.4) by setting $\left|r_{h e}\right|^{2} \simeq \Gamma^{2} / 4$ :

$$
g_{N S}^{c l}=\frac{N}{s+2 / \Gamma^{2}}
$$

For both the Orthogonal and Symplectic Ensembles the conductance is enhanced above the classical value, with a correction $O(N)$. Note in particular that in the absence of a field the conductance increases as disorder is added to the junction. When the length of the junction is such that $\Gamma s \simeq 1$, the junction conductance reaches its maximum value of roughly $g_{N S} \simeq N \Gamma / 2$. The classical value at this length is $g_{N S}^{c l} \simeq N \Gamma^{2} / 2$. This very large enhancement of the conductance is referred to as "reflectionless tunnelling."

In practice it is the sensitivity of the reflectionless tunnelling effect to a magnetic field which is observed in experiments [4]. We have seen explicitly in the analysis of the quasi-ballistic junction that the phase conjugation of electrons and holes traversing time reversed paths is destroyed in a magnetic field, and only the $O\left(N^{0}\right)$ correction, due to interference involving identical paths, should remain. Thus, by applying a magnetic field, the conductance of the junction should be dramatically reduced to its classical value, within a correction of $O(N)^{0}$. This argument also indicates that if we break spin rotation symmetry, the qualitative behaviour in a magnetic field should be unchanged, and there should only be quantitative corrections at $O\left(N^{0}\right)$ due to the spin orbit interaction. To confirm this we have carried out a numerical simulation of a junction under the relevant conditions.

The model used has been described in Section VII. The parameters used in the simulation are $L_{x}=L_{y}=6, L=48$ and $E_{F}=0$ for which $N=24$ at $\theta=0$ and $N=22$ at $\theta=\pi / 8$. We have set $W=2$ and estimate that the mean free path $l \simeq 12$. The junction is in the diffusive $\left(L_{z} \gg l\right)$ quasi- $1 d\left(L_{x}, L_{y} \leq l, L_{x}, L_{y} \ll L_{z}\right)$ regime. In Figure 6 we present the data for the conductance versus magnetic field obtained in the simulations for the two values of spin orbit interaction parameter $\theta$ indicated above. The magnetic field is given in terms of the flux $\phi$ penetrating the normal part of the junction. At $\mathrm{z}=\mathrm{L}$ there is a potential barrier of height $U_{0}=3$. The barrier transparency is a function of $U_{0}$ and also has a weak dependence on the the spin orbit parameter $\theta$ and the magnetic flux $\phi$. The average value of $\Gamma=0.23$ with a variation of about $10 \%$ over the parameter range used. At zero field and zero bias the conductance should be approximately:

$$
G_{N S} \simeq \frac{2 e^{2}}{h} \frac{N \Gamma}{2} \approx 5 \frac{e^{2}}{h}
$$

which is in reasonable agreement with the numerical data. On applying a magnetic field we expect to approach the classical value:

$$
G_{N S}^{c l}=\frac{2 e^{2}}{h} \frac{N}{s+2 / \Gamma^{2}} \approx \frac{e^{2}}{h}
$$

This is again in reasonable agreement with the numerical data. A strong reduction of the conductance is observed both when spin rotation symmetry is unbroken $\theta=0$ and when it is broken $\theta=\pi / 8$ by the spin orbit interaction. The critical field corresponds to a flux $\phi \simeq 2 \phi_{0}$ penetrating the sample, in agreement with critical field expected for the suppression of the weak localisation effect in normal metals. The data confirm that, apart from possible corrections of $O\left(N^{0}\right)$, the reflectionless tunnelling effect is not sensitive to the spin orbit interaction.

\section{CONCLUSIONS}

We have studied the effect of the breaking of spin rotation symmetry, as a result of an appreciable spin orbit interaction, in determining the quantum correction to the classical conductance of a disordered mesoscopic normal superconducting junction.

The most striking result we have obtained concerns the NS junction in an applied magnetic field. We have found that even if time reversal symmetry is broken, the quantum correction is sensitive to the spin orbit interaction. A semiclassical analysis of a quasi-ballistic junction permitted the scattering processes, which interfere to produce this effect, to be identified. They involve scattering paths in which an electron and a hole traverse a loop in the normal part of the junction in the same sense, that which we have called here "identical paths". 


\section{ACKNOWLEDGEMENTS}

Keith Slevin would like to thank the European Commission for financial support under the Human Capital and Mobility Programme. Pier Mello would like to thank the Wissenschaftskolleg zu Berlin for its hospitality, as well as the Service de Physique de l'État Condensé, CEA-Saclay, for the financial support given during his visit to Saclay in the early stages of this research. We would also like to thank Piet Brouwer for his helpful comments.

\section{APPENDIX A:}

In this appendix we group together certain technical details concerning the scattering theory for model (3.1) which appears in the main text. We adopt the Dirac notation so that, for example, $<x y z \sigma|\psi\rangle=\psi(x y z \sigma)$. The particle current $J_{p}(z)$ through a cross section at $z$ can be shown to be:

$$
J_{p}(z)=\frac{1}{i \hbar}<\psi|D[z]| \psi>
$$

where $D$ is the operator:

$$
\begin{aligned}
D[z]= & \sum_{x y \sigma \mu}|x y z \sigma><x y z \sigma| H_{e}|x y z+1 \mu><x y z+1 \mu| \\
& -|x y z+1 \sigma><x y z+1 \sigma| H_{e}|x y z \mu><x y z \mu|
\end{aligned}
$$

By rewriting the Schroedinger equation in the contact in terms of a real space transfer matrix, and considering the orthogonality relations between left and right eigenvectors of this matrix, it is possible to show that the Bloch states satisfy the orthogonality relation:

$$
<m|D[z]| n>=0 \text { if } k_{m} \neq k_{n}^{*}
$$

In addition for real $k$ the transverse wavefunctions may be normalised so that:

$$
< \pm m|D[z]| \pm n>= \pm i \delta_{n, m}
$$

Each channel then carrys a charge current of $e / \hbar$. Here we use the notation that:

$$
<x y z \sigma \mid \pm m>=\psi_{ \pm m}(x y \sigma) \exp \left( \pm i k_{m}\right)
$$

In the absence of a magnetic field the Hamiltonian $H_{e}$ will commute with the time reversal operator $\mathcal{T}$. In this case if $\psi^{e}$ is a solution to the scattering problem we may construct another solution $\mathcal{T} \psi^{\urcorner}$by operating on $\psi^{e}$ with $\mathcal{T}$. Taking note of this we can derive the following condition on $S_{e}$ if the Hamiltonian is time reversal invariant:

$$
S_{e}^{T}=\left[\begin{array}{cc}
d^{\dagger} & 0 \\
0 & -d^{\dagger}
\end{array}\right] S_{e}\left[\begin{array}{cc}
-d & 0 \\
0 & d
\end{array}\right]
$$

where $d$ is a $2 N \times 2 N$ symmetric unitary matrix with elements:

$$
d_{m, n}=-i \sum_{x, y, \sigma, \mu} \psi_{+m}^{*}\left(L_{x}-x+1, y, \sigma\right)\left[\left[v^{z \dagger}\right]_{\sigma, \mu} \exp \left(i k_{m}\right)-\left[v^{z}\right]_{\sigma, \mu} \exp \left(-i k_{n}\right)\right] \psi_{+n}^{*}(x, y, \mu)
$$

The condition on the scattering matrix when $H_{e}$ is time reversal invariant, can be simplified by an appropriate choice of basis. Since $d$ is symmetric unitary it may be decomposed (not uniquely) as $d=e^{T} e$ with $e$ unitary. If we do this and make a transformation to the new basis:

$$
\psi_{+n}^{\prime}=\sum_{m} e_{n, m} \psi_{+m}
$$

the matrix $d$ becomes unity $d=1_{2 N}$. This transformation is legitimate since it leaves the current normalisation unchanged. The general relation between the electron and hole scattering matrices arrived at is, as indicated in the main text: 


$$
S_{h}(+\epsilon,+B)=\left[\begin{array}{cc}
d^{T} & 0 \\
0 & -d^{T}
\end{array}\right] S_{e}^{*}(-\epsilon,+B)\left[\begin{array}{cc}
-d^{*} & 0 \\
0 & d^{*}
\end{array}\right]
$$

which again may be simplified by the transformation (A8).

We now turn to the relation between the electron scattering matrix $S_{e}$ and the electron Green function $G_{e}^{+}$. Let us suppose that $H=H_{e}+U$ with $U$ a random potential. To each incoming state at the left $\mid+n>$ we associate a scattering state $\mid \phi_{+n}>$ :

$$
\left|\phi_{+n}>=\right|+n>+G_{e}^{+} U \mid+n>
$$

Supposing that the random potential $U$ is nonzero only in the volume $\mathcal{V}$ between $z_{0}<z<z_{1}$ this can be transformed to:

$$
\begin{aligned}
\mid \phi_{+n}>= & G_{e}^{+} D\left[H_{e}, z_{1}\right]\left|+n>-G_{e}^{+} D\left[H_{e}, z_{0}-1\right]\right|+n> \\
& +\sum_{x y z \notin \mathcal{V} \sigma}|x y z \sigma><x y z \sigma|+n>
\end{aligned}
$$

In $z \leq z_{0}+1$ the scattering state has the form:

$$
\left|\phi_{+n}>=\right|+n>+\sum_{m}\left[r_{e}\right]_{m, n} \mid-m>
$$

and in the region $z \geq z_{1}-1$ :

$$
\left|\phi_{+n}>=\sum_{m}\left[t_{e}\right]_{m, n}\right|+m>
$$

With the aid of (A3) and (A4) we now find:

$$
\begin{aligned}
& {\left[r_{e}\right]_{m n}=-i<-m\left|D\left[z_{0}\right] G_{e}^{+} D\left[z_{0}-1\right]\right|+n>} \\
& {\left[t_{e}\right]_{m n}=+i<+m\left|D\left[z_{1}-1\right] G_{e}^{+} D\left[z_{0}-1\right]\right|+n>} \\
& {\left[r_{e}^{\prime}\right]_{m n}=-i<+m\left|D\left[z_{1}-1\right] G_{e}^{+} D\left[z_{1}\right]\right|-n>} \\
& {\left[t_{e}^{\prime}\right]_{m n}=+i<-m\left|D\left[z_{0}+1\right] G_{e}^{+} D\left[z_{1}\right]\right|-n>}
\end{aligned}
$$

The last two relations are obtained by considering an incoming wave $\mid-n>$ from the right. In a similar way a relation between $S_{h}$ and $G_{h}^{+}$can be derived. We note only the expression for the reflection matrix at the right:

$$
\left[r_{h}^{\prime}\right]_{m n}=-i<-m\left|D\left[z_{1}-1\right] G_{h}^{+} D\left[z_{1}\right]\right|+n>
$$

and we now assume that the magnetic field is zero except in $\mathcal{V}$.

\section{APPENDIX B:}

As mentioned in the text, the simplicity of (3.18) is related to the presence of $\rho$ in Eq.(3.5). In most references dealing with random matrix approaches to conduction in disordered solids definitions, such as those of ref. [16], $\rho$ does not appear in the equivalent of these equations. To avoid confusion we describe explicitly the transformation between the sets of definitions.

In the absence of a magnetic field, the scattering matrix of Ref. [16] which we shall call $S_{e}^{M P}$ (containing reflection and transmission matrices $r_{e}^{M P}, t_{e}^{M P}$, etc.) has the form:

$$
S_{e}^{M P}=\left[\begin{array}{ll}
r_{e}^{M P} & t_{e}^{\prime M P} \\
t_{e}^{M P} & r_{e}^{\prime M P}
\end{array}\right]
$$

and the property that:

$$
\left(S_{e}^{M P}\right)^{T}=\left[\begin{array}{cc}
Z^{T} & 0 \\
0 & Z^{T}
\end{array}\right] S_{e}^{M P}\left[\begin{array}{ll}
Z & 0 \\
0 & Z
\end{array}\right]
$$

where the $2 N \times 2 N$ antisymmetric unitary matrix $Z$ satisfies (C3) and (C4). The coefficients $a_{+}, a_{+}^{\prime}$ introduced in Section IV are identical to those of Ref. [16], while the coefficients $a_{-}, a_{-}^{\prime}$ are related by: 


$$
\begin{aligned}
& a_{-}^{M P}=Z a_{-} \\
& a_{-}^{\prime M P}=Z a_{-}^{\prime}
\end{aligned}
$$

The relation between $S$ matrices is then:

$$
S_{e}=\left[\begin{array}{cc}
Z^{T} & 0 \\
0 & 1
\end{array}\right] S_{e}^{M P}\left[\begin{array}{ll}
1 & 0 \\
0 & Z
\end{array}\right]
$$

so that (B2) and (B4) give the relation (3.9).

In the absence of spin-orbit coupling the two spin components would be independent and the reflection and transmission matrices would be block diagonal so that, in a suitable basis, $S^{M P}$ would have the structure:

$$
S_{e}^{M P}=\left[\begin{array}{cccc}
\tilde{r}_{e} & 0 & \tilde{t}_{e}^{\prime} & 0 \\
0 & \tilde{r}_{e} & 0 & \tilde{t}_{e}^{\prime} \\
\tilde{t}_{e} & 0 & \tilde{r}_{e}^{\prime} & 0 \\
0 & \tilde{t}_{e} & 0 & \tilde{r}_{e}^{\prime}
\end{array}\right]
$$

Here, $\tilde{r}_{e}, \tilde{t}_{e}, \ldots$ are $N \times N$ matrices that correspond to the reflection and transmission matrices for either spin direction and have the usual properties, so that $S_{e}^{M P}$ is symmetric. The $S$ of this paper, obtained from the transformation (B4), would have the structure:

$$
S_{e}=\left[\begin{array}{cccc}
0 & -\tilde{r}_{e} & \tilde{t}_{e}^{\prime} & 0 \\
\tilde{r}_{e} & 0 & 0 & \tilde{t}_{e}^{\prime} \\
\tilde{t}_{e} & 0 & 0 & \tilde{r}_{e}^{\prime} \\
0 & \tilde{t}_{e} & -\tilde{r}_{e}^{\prime} & 0
\end{array}\right]
$$

\section{APPENDIX C:}

The parameterisations of $S_{e}$ appropriate for the various ensembles are discussed in Refs. [3,16,24,33,34]. For the Unitary II Ensemble $S_{e}$ may be parameterised:

$$
S_{e}=\left[\begin{array}{cc}
u_{1} \sqrt{R} u_{3} & -u_{1} \sqrt{T} u_{4} \\
u_{2} \sqrt{T} u_{3} & u_{2} \sqrt{R} u_{4}
\end{array}\right]
$$

where the $u$ 's are $2 N \times 2 N$ unitary matrices, $T=\operatorname{diag}\left\{T_{n} ; n=1, \ldots, 2 N\right\}$ and $R=1_{2 N}-T$. (The parameters $T_{n}$ appearing here are the same as the eigenvalues of $t_{e} t_{e}^{\dagger}$ appearing in the conductance formulae of Section VI.) The unitary matrices are independently and uniformly distributed with respect to the invariant measure on the group $U(2 N)$ of $2 N \times 2 N$ unitary matrices.

In the Symplectic Ensemble, time reversal symmetry imposes further restrictions on the form of $S_{e}$ and the appropriate parameterisation is:

$$
S_{e}=\left[\begin{array}{cc}
u_{1} \sqrt{R} Z u_{1}^{T} & -u_{1} \sqrt{T} Z u_{2}^{T} \\
u_{2} \sqrt{T} Z u_{1}^{T} & u_{2} \sqrt{R} Z u_{2}^{T}
\end{array}\right]
$$

with $u_{1}$ and $u_{2}$ uniformly distributed on $U(2 N)$ and $Z$ is a fixed antisymmetric $2 N \times 2 N$ matrix satisfying:

$$
\begin{gathered}
{[Z, T]=0} \\
Z^{2}=-1_{2 N}
\end{gathered}
$$

If the spin orbit interaction is negligible the spin is conserved during the electron motion and the Hamiltonian and scattering matrices can be block diagonalised. Thus in the Unitary I Ensemble a basis can be found such that:

$$
S_{e}=\left[\begin{array}{cccc}
0 & -\tilde{u}_{1} \sqrt{\tilde{R}} \tilde{u}_{3} & \tilde{u}_{1} \sqrt{\tilde{T}} \tilde{u}_{4} & 0 \\
\tilde{u}_{1} \sqrt{\tilde{R}} \tilde{u}_{3} & 0 & 0 & \tilde{u}_{1} \sqrt{\tilde{T}} \tilde{u}_{4} \\
\tilde{u}_{2} \sqrt{\tilde{T}} \tilde{u}_{3} & 0 & 0 & -\tilde{u}_{2} \sqrt{\tilde{R}} \tilde{u}_{4} \\
0 & \tilde{u}_{2} \sqrt{\tilde{T}} \tilde{u}_{3} & \tilde{u}_{2} \sqrt{\tilde{R}} \tilde{u}_{4} & 0
\end{array}\right]
$$


where $\tilde{u}_{1}, \ldots, \tilde{u}_{4}$ are $N \times N$ unitary matrices uniformly distributed on the unitary group $U(N)$ and $\tilde{T}=\operatorname{diag}\left\{\tilde{T}_{n} ; n=\right.$ $1, \ldots, N\}$ and $\tilde{R}=1_{N}-\tilde{T}$.

Finally in the Orthogonal ensemble $S_{e}$ is parameterised:

$$
S_{e}=\left[\begin{array}{cccc}
0 & -\tilde{u}_{1} \sqrt{\tilde{R}} \tilde{u}_{1}^{T} & \tilde{u}_{1} \sqrt{\tilde{T}} \tilde{u}_{2}^{T} & 0 \\
\tilde{u}_{1} \sqrt{\tilde{R}} \tilde{u}_{1}^{T} & 0 & 0 & \tilde{u}_{1} \sqrt{\tilde{T}} \tilde{u}_{2}^{T} \\
\tilde{u}_{2} \sqrt{\tilde{T}} \tilde{u}_{1}^{T} & 0 & 0 & -\tilde{u}_{2} \sqrt{\tilde{R}} \tilde{u}_{2}^{T} \\
0 & \tilde{u}_{2} \sqrt{\tilde{T}} \tilde{u}_{1}^{T} & \tilde{u}_{2} \sqrt{\tilde{R}} \tilde{u}_{2}^{T} & 0
\end{array}\right]
$$

with $\tilde{u}_{1}$ and $\tilde{u}_{2}$ uniformly distributed on $U(N)$.

The distribution of the $T_{n}$ 's is given by the solution of a Fokker Planck equation, the precise form of which depends on the ensemble under consideration. The joint distribution, and in particular the correlations, of the $T_{n}$ 's depend on the symmetry of the ensemble under consideration. However, all we need to know for the present purpose is that: $[35,36]$

$$
\left\langle\sum_{n=1}^{2 N} T_{n}\right\rangle=2 N(1-s)+O\left(s^{2}\right)
$$

independent of the ensemble. The only further information needed to carry out the required averages is that if $u$ is an $N \times N$ unitary matrix uniformly distributed on $U(N)$ then

$$
<u_{i, j} u_{i^{\prime}, j^{\prime}}^{*}>=\frac{1}{N} \delta_{i, i^{\prime}} \delta_{j, j^{\prime}}
$$

An important property of the local maximum entropy model is that the distribution of $S_{e}$ is isotropic, that is to say the matrices $u_{1}, \ldots, u_{4}$ are distributed according to the invariant measure on the unitary group and independently of the parameters $T_{1}, \ldots, T_{2 N}$.

[1] G. Bergmann, Physics Reports 107, 1 (1984.)

[2] G. Bergmann, Solid State Communications 42, 815 (1982.)

[3] K. Slevin and T. Nagao, International Journal of Modern Physics B 9, 103 (1995.)

[4] A. Kastalsky, A. Kleinsasser, L. Greene, R. Bhat, F. Milliken and J. Harbison, Physical Review Letters 67, 3026 (1991.)

[5] B. van Wees, P. de Vires, P. Magnee and T. Klpawijk, Physical Review Letters 69, 510 (1992.)

[6] The random phase or isotropy assumption for the scattering matrix is described in Appendix C and in Refs. [3,29,30]

[7] Y. Takane and H. Otani, Journal of the Physical Society of Japan 63, 3361 (1994.)

[8] C. W. J. Beenakker, Physical Review B 49, 2205 (1994.)

[9] C. Beenakker in Mesoscopic Quantum Physics, edited by E. Akkermans, G. Montambaux, J-L Pichard and J. Zinn-Justin (North-Holland, Amsterdam, to be published.)

[10] P. Brouwer and C. Beenakker, Physical Review B,52, R3868 (1995.)

[11] P. de Gennes, Superconductivity of Metals and Alloys, (Benjamin, New York, 1966.)

[12] T. Ando and H. Tamura, Physical Review B 46, 2332 (1992.)

[13] G. Arfken, Mathematical Methods for Physicists, (Academic Press, New York 1970.)

[14] N. Zanon and J-L. Pichard, Journal de Physique France 49, 1387 (1988.)

[15] S. N. Evangelou and T. Ziman, Journal of Physics C 20, L235 (1987.)

[16] P. Mello and J-L Pichard, Journal de Physique 1 France 1, 493 (1991.)

[17] A. F. Andreev, Soviet Physics JETP 19, 1228 (1964.)

[18] H. Feshbach, Topics in the Theory of Nuclear Reactions, in Reaction Dynamics (Gordon and Breach, New York, 1973).

[19] G. E. Blonder, M. Tinkham and T. M. Klapwijk, Physical Review B 25, 4515 (1982.)

[20] C. Lambert, Journal of Physics: Condensed Matter 3, 6579 (1991.)

[21] Y. Takane and H. Ebisawa, Journal of the Physical Society of Japan 61, 1685 (1992.)

[22] For a diffusive system the Thouless energy is $E_{c}=\hbar D / L^{2}$ where $D$ is the diffusion constant and $L$ a typical dimension of the sample. For a ballistic sample $E_{c}=\hbar v_{F} / L$ where $v_{F}$ is the Fermi velocity. 
[23] C. Beenakker, Physical Review B 46, 12841, (1992.)

[24] P. Mello, P. Pereyra and N. Kumar, Annals of Physics 181, 290 (1988.)

[25] A. D. Stone, P. A. Mello, K,A. Muttalib and J-L. Pichard, in Mesoscopic Phenomena in Solids, edited by B. L. Altshuler, P.A. Lee and R. A. Webb (North Holland, Amsterdam, 1991.)

[26] K. Frahm, Physical Review Letters 74, 4706 (1995.)

[27] P. Brouwer and K. Frahm, to appear in Phys. Rev. B.

[28] M. Gutzwiller, Chaos in Classical and Quantum Mechanics, (Spring Verlag, New York 1990.)

[29] K. Slevin, J-L. Pichard and K. A. Muttalib, Journal de Physique 1 France 3, 1387 (1993.)

[30] R. A. Jalabert and J-L. Pichard, Journal de Physique 1 France 5, 287 (1995.)

[31] P. A. Mello and J.-L. Pichard, Physical Review B 40, 5276 (1989.)

[32] C. Beenakker, B. Rejaei and J. Melsen, Physical Review Letters 72, 2470 (1994.)

[33] H. U. Baranger and P. A. Mello, Physical Review Letters 73, 142 (1994)

[34] R. A. Jalabert, J.-L. Pichard and C. W. J. Beenakker, Europhysics Letters 27, 255 (1994.)

[35] P. Mello and A. Stone, Physical Review B 44, 3559 (1991.)

[36] A. Macedo and J. Chalker, Physical Review B 46, 14985 (1992.) 
Figure captions:

Figure 1: A schematic of the NS junction for which the scattering theory is developed in Section III.

Figure 2: An example of a path which contributes to process $p_{1}$ corresponding to an electron (solid line) traversing the normal part of the junction whose path is then retraced by the Andreev reflected hole (dashed line).

Figure 3: An example of a "time reversed path" which contributes to process $p_{5}$. The path of the electron moving from $x_{a}$ to $x_{b}$ is retraced by the Andreev reflected hole as it moves from $x_{b}$ to $x_{a}$. Interference between this path and that illustrated in Figure 2 is insensitive to the spin orbit interaction and is suppressed in a magnetic field.

Figure 4: An example of an "identical path" which contributes to the process $p_{5}$. The electron moves around a loop and returns to $x_{a}$. The Andreev reflected hole traverses the loop in the same direction. Interference between this path and that illustrated in Figure 2 is sensitive to the spin orbit interaction but not to a magnetic field.

Figure 5: The conductance of a quasi-1d diffusive NS junction as a function of the spin orbit interaction parameter $\theta$ in the presence of an applied magnetic field ( flux of $1 / 25 \phi_{0}$ per lattice cell). The barrier transparency $\Gamma=1$. The NS conductance is sensitive to the breaking of spin rotation symmetry even though time reversal symmetry is broken, while the normal conductance is approximately constant as expected.

Figure 6: The conductance of a quasi-1d NS junction as a function of magnetic flux penetrating the normal part of the junction for zero $\theta=0$ and strong $\theta=\pi / 8$ spin orbit interaction. The barrier transparency is low $(\Gamma \simeq 0.23)$ and the length of the junction is such that $\Gamma s \simeq 1$. We see that the qualitative features of the reflectionless tunnelling effect are insensitive to the spin orbit interaction.

TABLE I. The various ensembles for which $G_{N S}$ is calculated. The abbreviation TRS means time reversal symmetry and SRS spin rotation symmetry.

\begin{tabular}{|l|l|l|}
\hline \hline Ensemble & TRS & SRS \\
\hline Orthogonal & yes & yes \\
Unitary I & broken & yes \\
Unitary II & broken & broken \\
Symplectic & yes & broken \\
\hline \hline
\end{tabular}

TABLE II. The quantum correction $\delta G_{N S}=\left(2 e^{2} / h\right) \delta g_{N S}$ for the quasi-ballistic NS junction for the ensembles listed in Table 1.

\begin{tabular}{|l|l|l|l|}
\hline \hline Ensemble & $\delta g_{N S}$ & $\Gamma=1$ & $\Gamma \ll 1$ \\
\hline Orthogonal/Symplectic & $-2 N s f(\Gamma)$ & $-4 N s$ & $+N s \Gamma^{2}$ \\
Unitary I & $-2 s f(\Gamma)$ & $-4 s$ & $+s \Gamma^{2}$ \\
Unitary II & $+s f(\Gamma)$ & $+2 s$ & $-s \Gamma^{2} / 2$ \\
\hline \hline
\end{tabular}

TABLE III. The quantum correction $\delta G_{N S}=\left(2 e^{2} / h\right) \delta g_{N S}$ for a diffusive $(1 \ll s \ll N)$ NS junction without a Schottky barrier $(\Gamma=1)$.

\begin{tabular}{|l|ll|}
\hline \hline Ensemble & $\delta g_{N S}$ & $(-0.593)$ \\
\hline Orthogonal & $\frac{4}{\pi^{2}}-1$ & $(+0.297)$ \\
Symplectic & $-\frac{1}{2}\left(\frac{4}{\pi^{2}}-1\right)$ & $(-0.333)$ \\
Unitary I & $-\frac{1}{3}$ & $(+0.166)$ \\
Unitary II & $+\frac{1}{6}$ & \\
\hline \hline
\end{tabular}




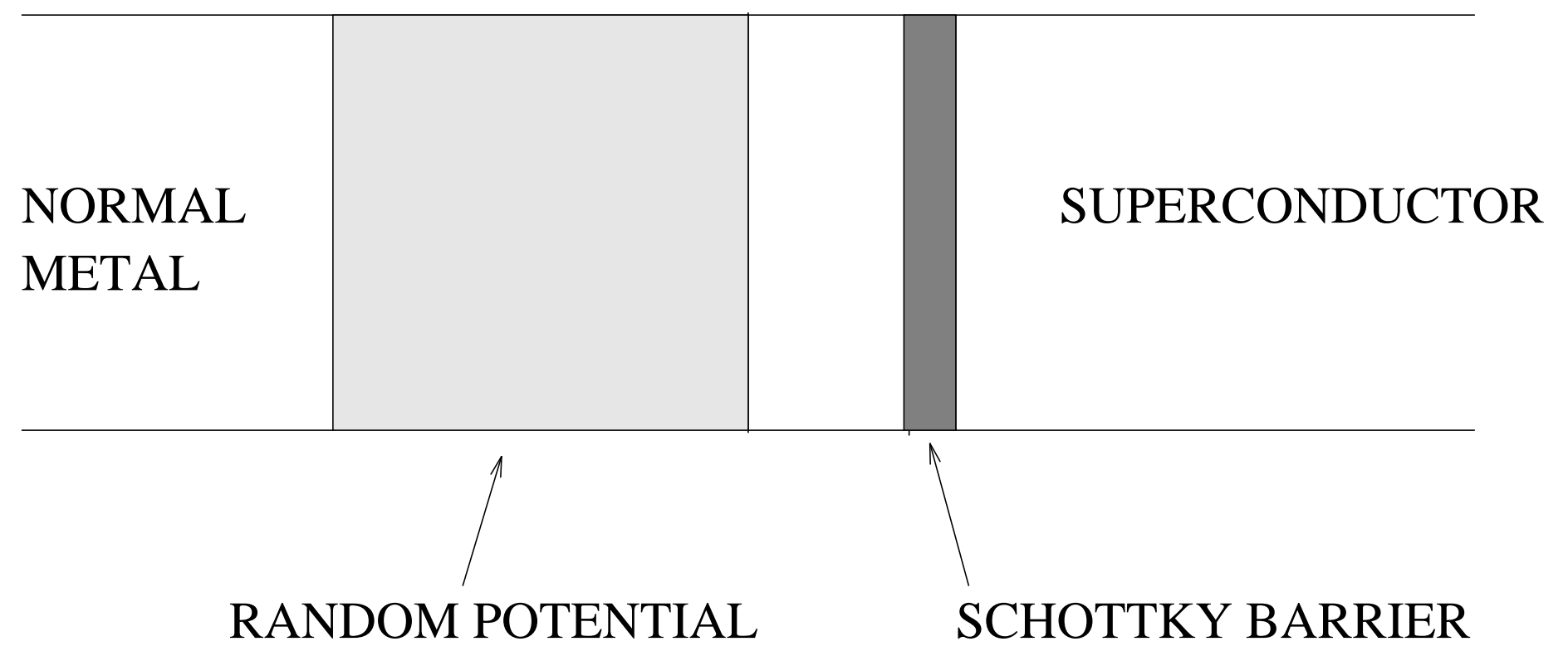




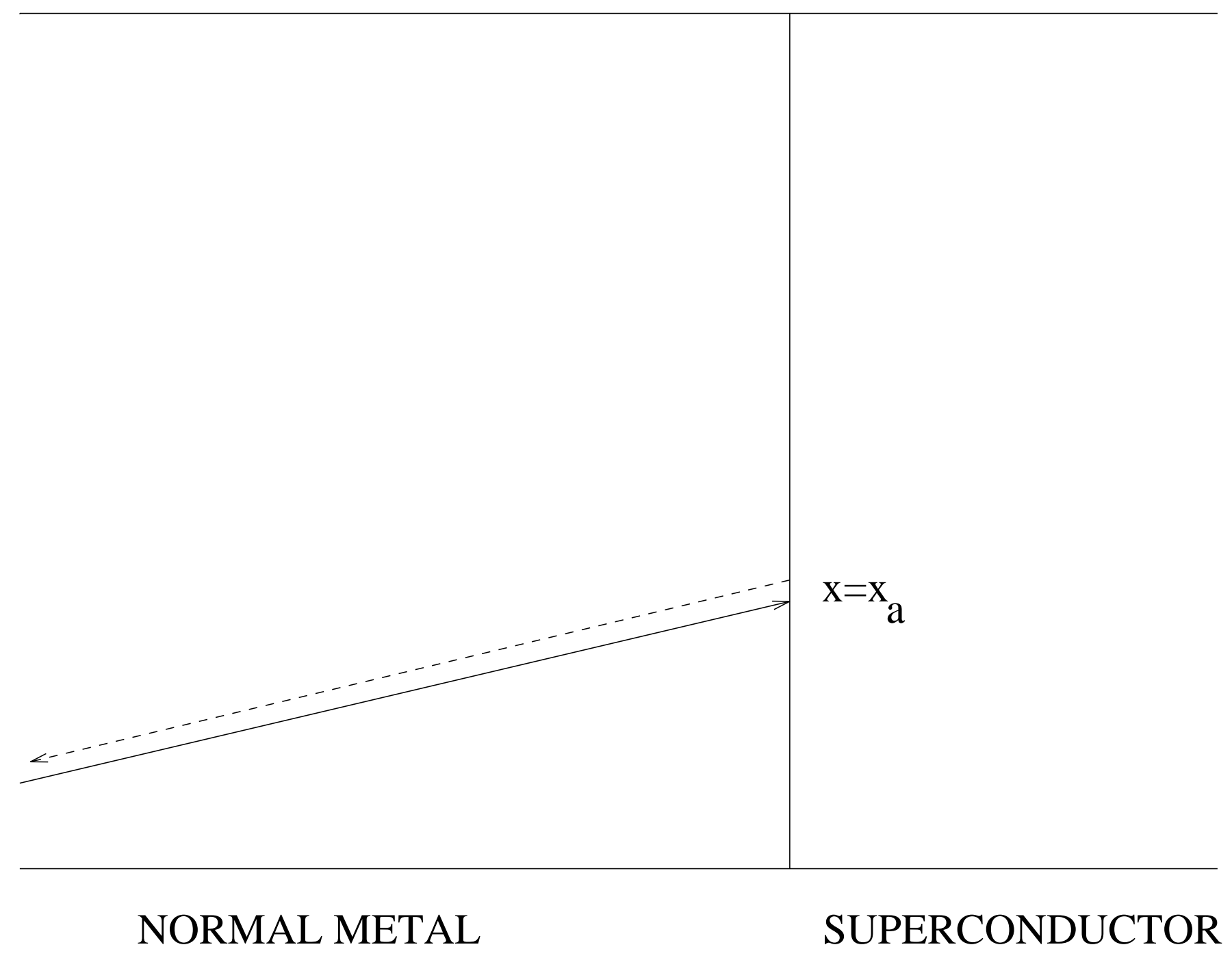




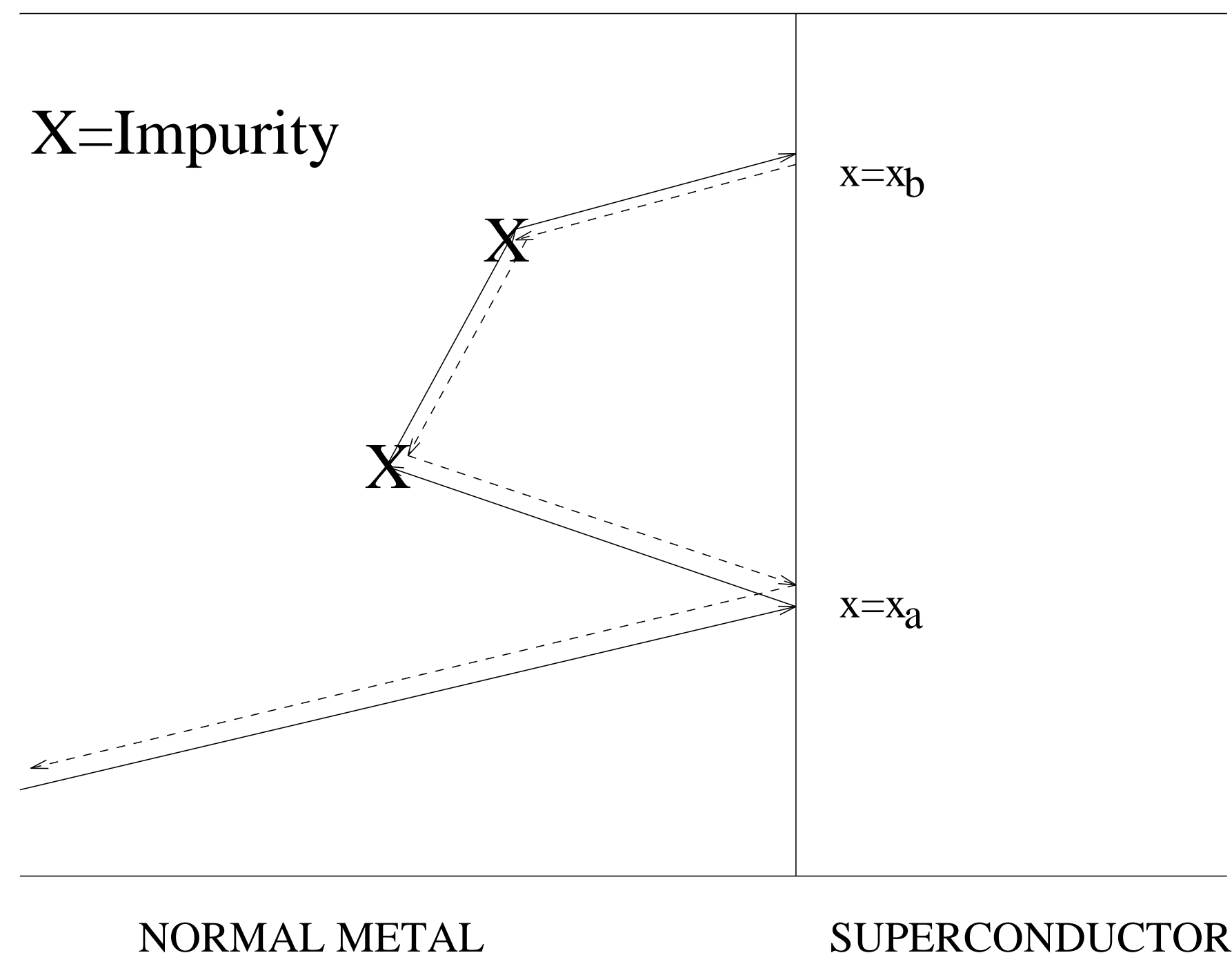




\section{$\mathrm{X}=$ Impurity}

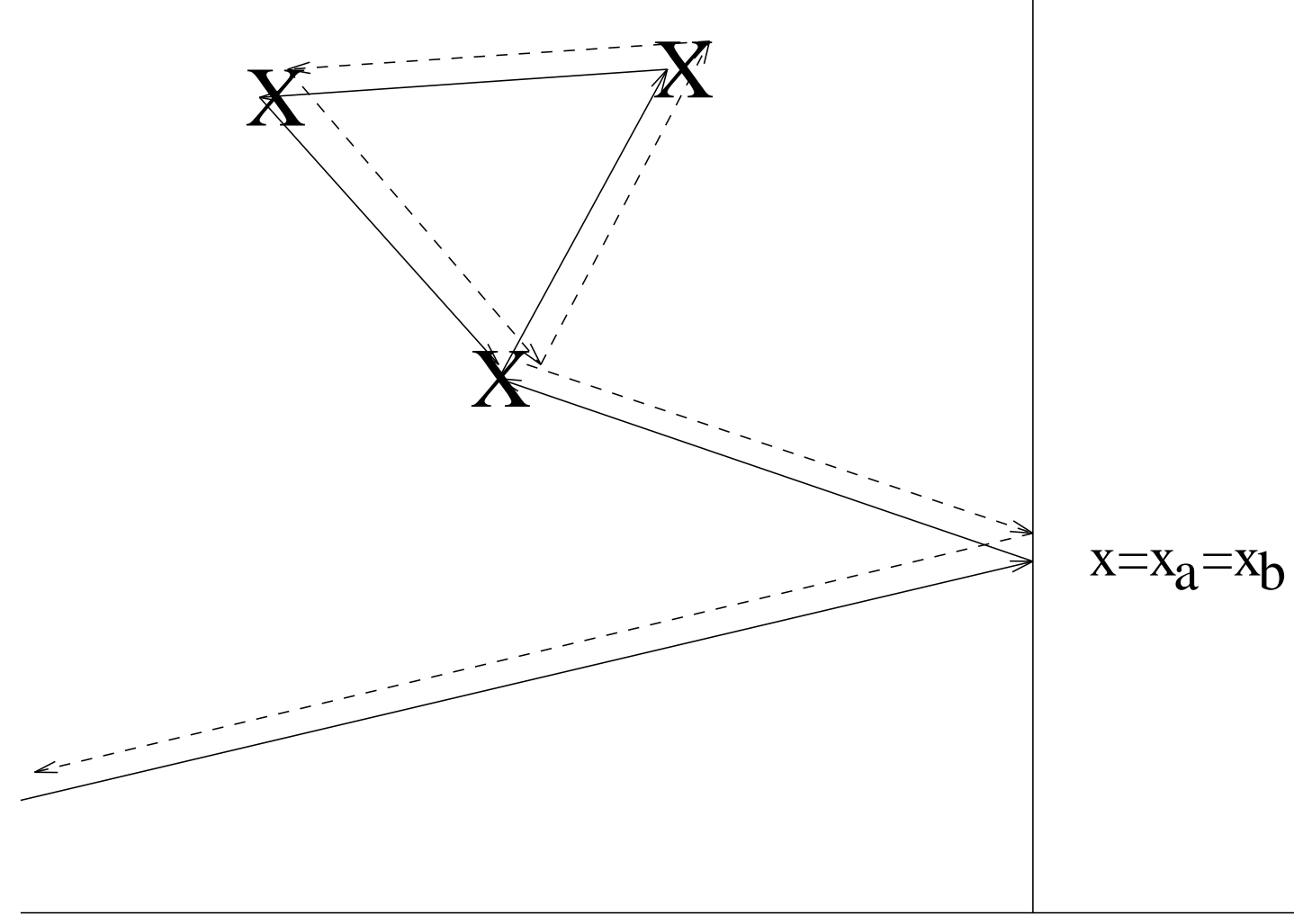

NORMAL METAL 


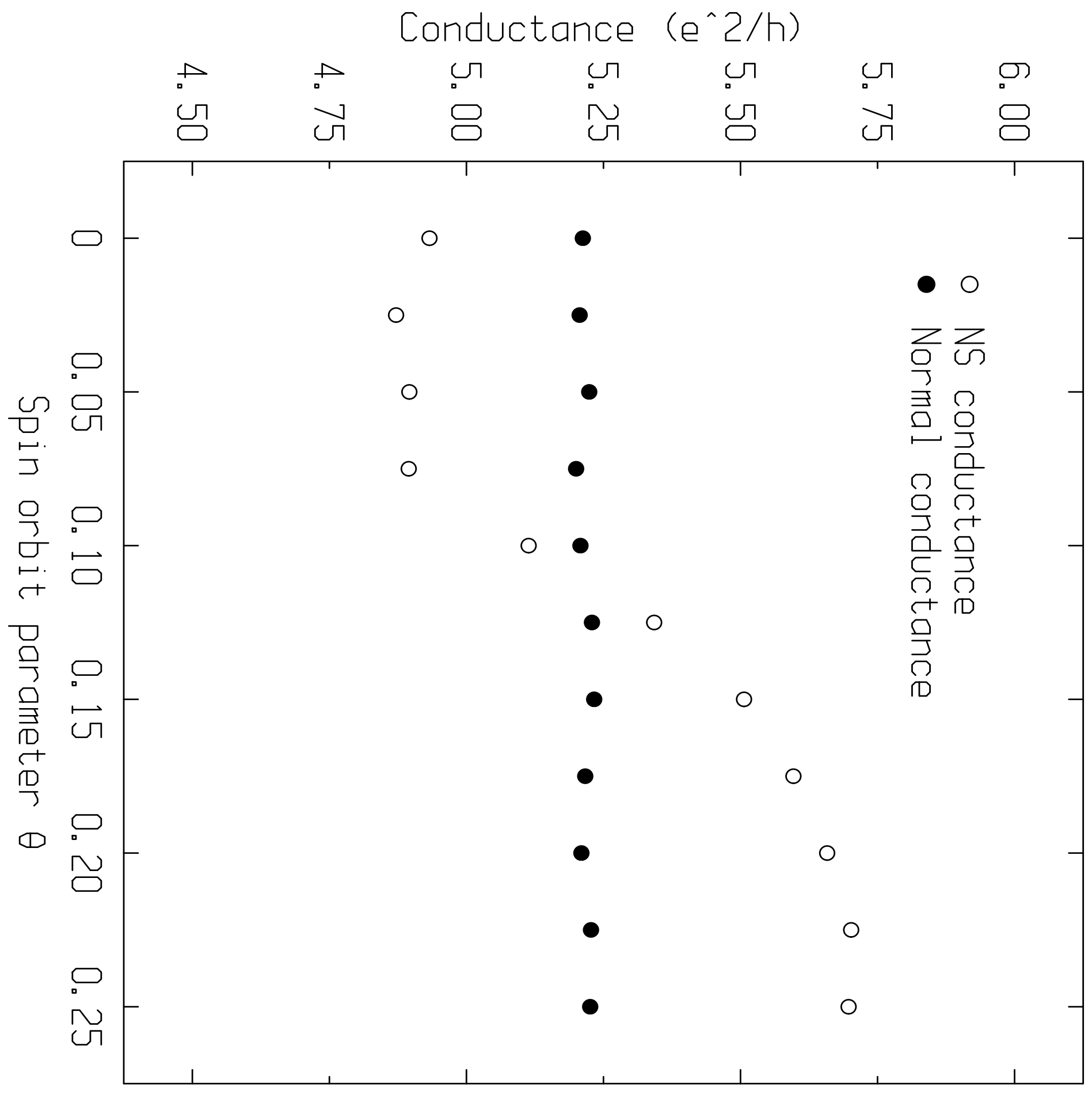




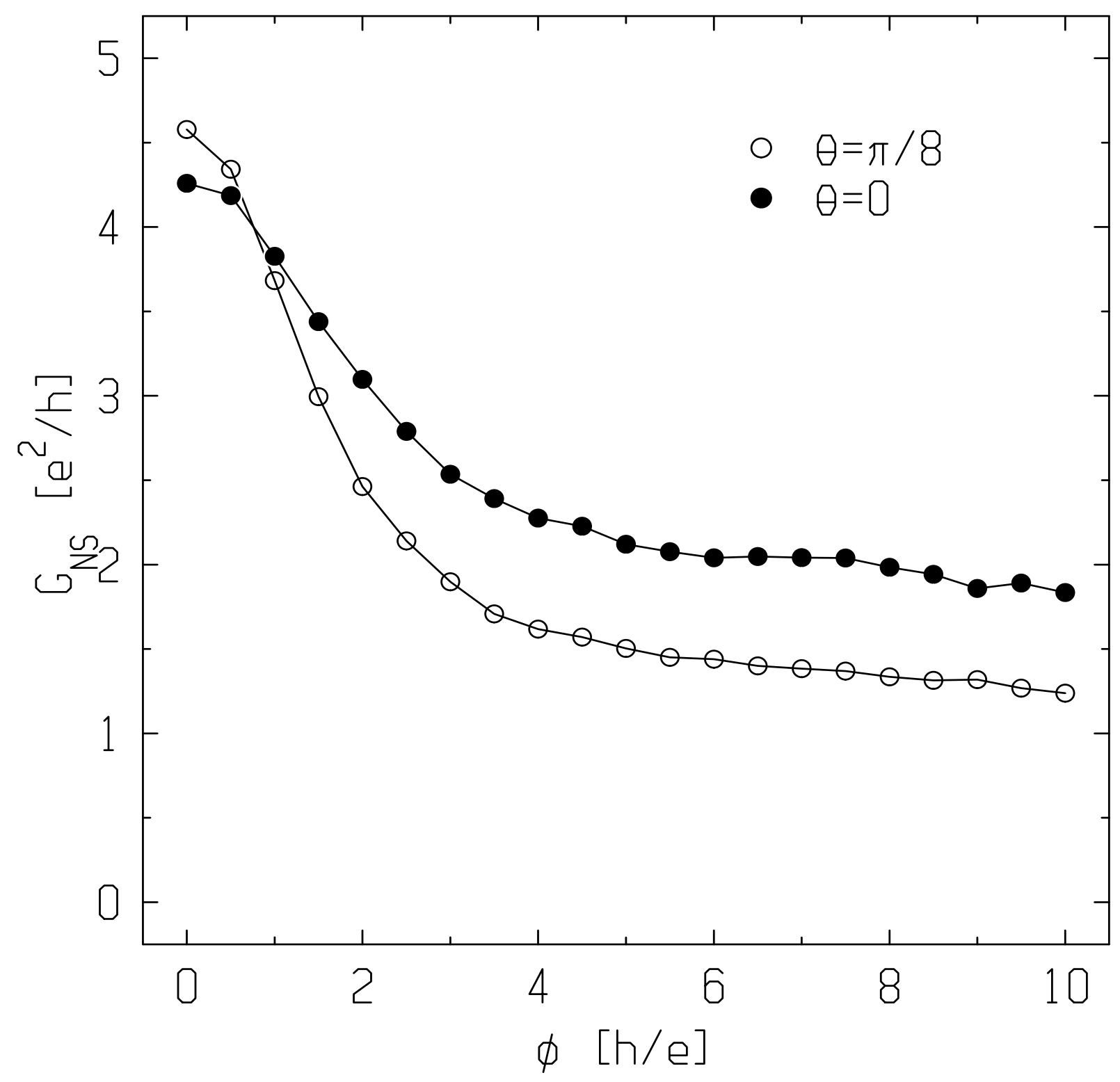

